\title{
Veronesean representations of projective spaces over quadratic associative division algebras
}
A. De Schepper
O. Krauss
J. Schillewaert
H. Van Maldeghem

\begin{abstract}
We provide a common description and geometric characterization of the Veronesean representations of all projective spaces defined over finite-dimensional quadratic alternative division algebras. We also study the homogeneity of these representations, and the irreducibility and indecomposability of the induced projective representations of the corresponding simple (automorphism) groups.
\end{abstract}

\section{Introduction}

It is well known that, to every quadratic alternative division algebra $\mathbb{A}$ over the field $\mathbb{K}$, corresponds a Veronesean representation of the projective plane associated with $\mathbb{A}$ in a projective space over $\mathbb{K}$ of dimension $3 d+2$, where $d=\operatorname{dim}_{\mathbb{K}} \mathbb{A}$ (see for instance [9]). Such Veronesean representations realise in a geometric way the Tits indices ${ }^{2}\left(A_{2} \times A_{2}\right)_{2}^{(1)}, A_{5,2}^{(2)}$ and $E_{6,2}^{28}$ of real forms of simple split algebraic groups, thus providing homogeneous descriptions of the corresponding geometries. Mazzocca \& Melone [11] proposed in 1984 an axiom system for Veronesean representations of projective spaces in case $\mathbb{K}=\mathbb{A}$ is a finite field. The advantage of that axiom system is that, if suitably extended, it can be thought of as a "functor" from the class of quadrics to the class of Veronesean representations of certain Tits-buildings, and this without presupposing the "output" geometries. To be more explicit, it is not built in the axioms which geometries one aims to characterise; they only state some properties of the Veronesean representations as differential varieties in the real and complex case (see below). The axioms gave rise to much classification and characterization work. The sets satisfying the axioms of Mazzocca \& Melone are usually called Veronesean caps, although they are really only "caps" if the input quadrics have Witt index 1. Also, one distinguishes between global and local Veronesean caps. The former model the geometries of the second row of the Freudenthal-Tits magic square, and the latter also include the higher rank analogues of that second row. The ultimate goal is to classify all Veronesean caps. If we restrict ourselves to the case where we really have caps, hence where the input is a quadric of Witt index 1, or more generally, an ovoid in a projective space (as introduced by Tits [17]), then the finite case was completely settled by Mazzocca \& Melone [11], Hirschfeld \& Thas [6], Thas \& Van Maldeghem [16], and Cooperstein, Thas \& Van Maldeghem [4]. In the general case, partial results were obtained by Schillewaert \& Van Maldeghem [12,13] and Krauss [8], and a complete classification of all global Veronesean caps 
was achieved recently by Krauss, Schillewaert \& Van Maldeghem [9]. Besides the ordinary quadric Veroneseans and the Veronesean representations of the projective planes related to the Tits indices mentioned above, a new kind of Veronesean was found, corresponding to a purely inseparable extension $\mathbb{A}$ of the field $\mathbb{K}$ in characteristic 2 such that $\mathbb{K}$ contains all squares of $\mathbb{A}$. In the latter case, the dimension of the ambient projective space could even be infinite. Also, in that case, the "functoriality" of the Mazzocca-Melone axioms was illustrated by the fact that a lot of properties of the input quadrics carry over to the output geometry, such as the existence of a large nucleus and the indecomposability and reducibility of the projective representation of the corresponding linear group.

In the present paper, we settle the finite-dimensional case for quadrics of Witt index 1. In particular:

- We classify the local non-global Veronesean caps embedded in a finite-dimensional projective space, say defined over the field $\mathbb{K}$, by showing that, for each such cap $\mathscr{K}$, there exists an associative quadratic division algebra $\mathbb{A}$ over $\mathbb{K}$ and an integer $n \geq 3$ such that $\mathscr{K}$ is a projection of the so-called standard Veronesean cap $\mathscr{V}_{n}(\mathbb{K}, \mathbb{A})$. The latter is the representation of the $n$-dimensional projective space $\mathscr{P}^{n}(\mathbb{A})$ in a projective space $\mathbb{P}(V)$ of dimension $n+\frac{n(n+1)}{2} \operatorname{dim}_{\mathbb{K}} \mathbb{A}$ over $\mathbb{K}$ as the image under the Veronesean map.

- Denoting by $\mathrm{PSL}_{n+1}(\mathbb{A})$ the little projective group of $\mathscr{P}^{n}(\mathbb{A})$ (i.e., the group generated by all root elations), we show that $\operatorname{PSL}_{n+1}(\mathbb{A})$ is induced in $\mathscr{V}_{n}(\mathbb{K}, \mathbb{A})$ by $\operatorname{PSL}(V)$, with above notation. Let $G^{\dagger}$ be the corresponding subgroup of $\operatorname{PSL}(V)$.

- We show that $G^{\dagger}$ acts irreducibly on $\mathbb{P}(V)$ except when $\mathbb{A}=\mathbb{K}$ with char $\mathbb{K}=2$, and except when $\mathbb{A}$ is a purely inseparable extension of $\mathbb{K}$, and reducibly otherwise. We determine all invariant subspaces and show that the representation is nevertheless indecomposable. We also describe the action of $G^{\dagger}$ on the invariant subspaces (this is only relevant in the purely inseparable case, where the action is defined by a line Grassmannian).

- Finally, we show that the only "homogeneous" local Veronesean caps (meaning, those admitting $\operatorname{PSL}_{n+1}(\mathbb{A})$ induced from the ambient projective space) are the standard local Veronesean caps.

Our proofs rely on the classification of the global case [9] (and the global Veronesean caps are subcaps of the local ones), and uses ideas from [4]. However, the existence and appearance of the purely inseparable case complicates things drastically. Indeed, with below notation and terminology, it is enough, in the 'regular' case, to "make all hyperplanes full", see Lemma 4.9 below. For the inseparable case, this does not suffice as the subspace from which one can project could be entirely situated in the nucleus subspace. This requires new arguments compared to [4]. It should also be mentioned that some typical counting arguments of the latter paper had to be replaced with other, more geometric, reasonings. This is not always straightforward. Our proof holds in general and hence provides alternative arguments for the finite case.

The application to projective representations of the corresponding little projective groups is new in all cases. The main achievement here is that we can show that an element $g$ of $G^{\dagger}$ (with above notation) is induced in the projection of $\mathscr{V}_{n}(\mathbb{K}, \mathbb{A})$ from a subspace $S$ if and only if $g$ stabilizes $S$ (the 'if'-part being trivial). 


\section{Definitions and Statement of the Main Results}

\subsection{Definition of a local Veronesean cap}

An ovoid $O$ in a possibly infinite-dimensional projective space $\Sigma$ is a set of points of $\Sigma$ such that no line of $\Sigma$ intersects $O$ more than 2 points, and for every point $x \in O$, there is a unique hyperplane $\pi$ through $x$ intersecting $O$ in only $x$ and containing all lines through $x$ that meet $O$ in only $x$. The hyperplane $\pi$ is called the tangent hyperplane at $x$ to $O$ and denoted $T_{x}(O)$. The dimension of the ovoid is equal to $-1+\operatorname{dim} \Sigma$.

Let $V$ be a possibly infinite-dimensional right vector space over some skew field $\mathbb{K}$, and let $\mathbb{P}(V)$ be the corresponding projective space. Let $X$ be a spanning point set of $\mathbb{P}(V)$ and let $\Xi$ be a collection of subspaces of $\mathbb{P}(V)$, which we shall refer to as the elliptic spaces of $X$, such that, for any $\xi \in \Xi$, the intersection $\xi \cap X$ is an ovoid $X(\xi)$ in $\xi$ of dimension at least 1 (and then, for $x \in X(\xi)$, we sometimes denote $T_{x}(X(\xi))$ simply by $T_{x}(\xi)$ ). We call $(X, \Xi)$, or briefly $X$, a local Veronesean cap if the following properties (V1), (V2) and (V3) hold.

(V1) Any two points $x$ and $y$ of $X$ lie in a unique element of $\Xi$, denoted by $[x, y]$.

(V2) If $\xi_{1}, \xi_{2} \in \Xi$, with $\xi_{1} \neq \xi_{2}$, then $\xi_{1} \cap \xi_{2} \subset X$.

(V3) For every $x \in X$, every $\xi \in \Xi$, with $x \notin \xi$, and every triple of distinct points $y_{1}, y_{2}, y_{3} \in$ $X(\xi)$, we have $T_{x}\left(\left[x, y_{3}\right]\right) \subseteq\left\langle T_{x}\left(\left[x, y_{1}\right]\right), T_{x}\left(\left[x, y_{2}\right]\right)\right\rangle$.

If, instead of (V3), the following stronger axiom (V3*) holds, then we speak of a global Veronesean cap.

(V3*) For every $x \in X$ and every every triple $\xi_{1}, \xi_{2}, \xi_{3} \in \Xi$, with $x \in \xi_{1} \cap \xi_{2} \cap \xi_{3}$, we have $T_{x}\left(\xi_{1}\right) \subseteq\left\langle T_{x}\left(\xi_{2}\right), T_{x}\left(\xi_{3}\right)\right\rangle$.

Ovoids which are the intersection of an elliptic space with $X$ are called $X$-ovoids. If $V$ is finitedimensional, then we say that $X$ is a finite-dimensional local or global Veronesean cap. If all $X$-ovoids have the same dimension $k$, then we say that $X$ has subdimension $k$.

Lemma 2.1 Every local Veronesean cap $(X, \Xi)$ admits a subdimension.

Proof The same proof as for the global case holds for local Veronesean caps, see (the proof of) Lemma 4.1 of [9].

For $x \in X$ and $x \notin \xi \in \Xi$, we denote by $T(x, \xi)$ the subspace of $\mathbb{P}(V)$ generated by all $T_{x}(\phi)$, for $x \in \phi$ and $\phi \cap \xi \neq \emptyset$. Axiom (V3) implies that $T(x, \xi)=\left\langle T_{x}\left(\phi_{1}\right), T_{x}\left(\phi_{2}\right)\right\rangle$, for every pair $\phi_{1}, \phi_{2} \in \Xi$ with $\phi_{i} \cap \xi \neq \emptyset, i=1,2$, and $\phi_{1} \neq \phi_{2}$. 


\subsection{Standard local Veronesean caps}

We now present a class of examples of local Veronesean caps. Trivially, all global Veronesean caps are local Veronesean caps. But there are additional examples, which we now present.

Let $\mathbb{A}$ be a quadratic associative division algebra over the field $\mathbb{K}$, where we assume $\mathbb{K} \subseteq \mathbb{A}$. This means that $\mathbb{A}$ is a skew field, $\mathbb{K}$ is a subfield of the center of $\mathbb{A}$ and every element $x \in \mathbb{A}$ satisfies a quadratic equation with coefficients in $\mathbb{K}$. Recall that one of the following situations holds.

- $\mathbb{A}=\mathbb{K}\left(\right.$ and $\left.\operatorname{dim}_{\mathbb{K}} \mathbb{A}=1\right)$;

- $\mathbb{A}$ is a quadratic Galois extension of $\mathbb{K}\left(\right.$ and $\operatorname{dim}_{\mathbb{K}} \mathbb{A}=2$ );

- $\mathbb{A}$ is a quaternion division algebra with center $\mathbb{K}\left(\right.$ and $\left.\operatorname{dim}_{\mathbb{K}} \mathbb{A}=4\right)$;

- $\mathbb{A}$ is a purely inseparable extension of $\mathbb{K}$ in characteristic 2 such that $\mathbb{K}$ contains the squares of all elements of $\mathbb{A}$ (and either $\operatorname{dim}_{\mathbb{K}} \mathbb{A}$ is infinite or $\operatorname{dim}_{\mathbb{K}} \mathbb{A}=2^{\ell}$, with $\ell \geq 2$ ).

In each case there exists a unique involution $\sigma$ on $\mathbb{A}$, which is an anti-automorphism (i.e., $(x y)^{\sigma}=y^{\sigma} x^{\sigma}$, for all $\left.x, y \in \mathbb{A}\right)$, which is the identity on $\mathbb{K}$ and for which both $a+a^{\sigma}$ and $a a^{\sigma}$ belong to $\mathbb{K}$ (and we call $\sigma$ the standard involution). In fact, every $a \in \mathbb{A}$ then satisfies the quadratic equation $x^{2}-\left(a+a^{\sigma}\right) x+a^{\sigma} a=0$.

Let $n$ be a natural number not smaller than 2 . We define the following vector space over $\mathbb{K}$.

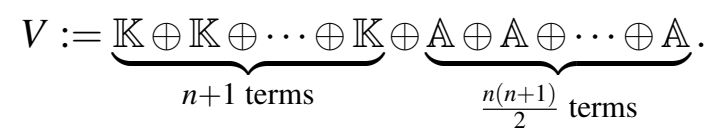

We can label the first $n+1$ terms with $0,1, \ldots, n$, and the other ones with $(j, \ell), j, \ell \in\{0,1, \ldots, n\}$, $j<\ell$. Consider $\mathbb{A}^{n+1}$ as an $(n+1)$-dimensional right vector space over $\mathbb{A}$. For every 1 -space $\left(x_{0}, x_{1}, \ldots, x_{n}\right) \mathbb{A} \in \mathbb{A}^{n+1}$ we define the 1-space $\mathbb{K}\left(y_{i}, y_{j, \ell}\right)_{0 \leq i \leq n, 0 \leq j<\ell \leq n}$ of $V$ as follows.

- $y_{i}=x_{i} x_{i}^{\sigma}, 0 \leq i \leq n$;

- $y_{j, \ell}=x_{j} x_{\ell}^{\sigma}, 0 \leq j<\ell \leq n$.

This is well-defined since right multiplication in $\mathbb{A}^{n+1}$ with some $a \in \mathbb{A}$ results in multiplication of the $y_{i}$ and the $y_{j, \ell}$ with $a a^{\sigma} \in \mathbb{K}$. Let $X$ be the corresponding set of points of $\mathbb{P}(V)$. Note that $X$ is the standard Veronesean embedding of the $n$-dimensional projective space $\mathscr{P}^{n}(\mathbb{A})$ over $\mathbb{A}$ when $\mathbb{A}$ is not an inseparable extension of $\mathbb{K}$, and it yields a new type of Veronesean embedding when $\mathbb{A}$ is an inseparable extension of $\mathbb{K}$ in characteristic 2 . The points of $\mathscr{P}^{n}(\mathbb{A})$ are in bijective correspondence with the elements of $X$. Every line of $\mathscr{P}^{n}(\mathbb{A})$ corresponds to a point set in $X$ isomorphic to a quadric of Witt index 1 that spans a subspace of dimension $1+\operatorname{dim}_{\mathbb{K}} \mathbb{A}$. If we define these subspaces to be the elliptic subspaces, then we will show in Theorem 3.3 below that $X$ is a local Veronesean cap of subdimension $\operatorname{dim}_{\mathbb{K}} \mathbb{A}$. We denote it by $\mathscr{V}_{n}(\mathbb{K}, \mathbb{A})$ and call it the standard local Veronesean cap of index $n$ associated with $(\mathbb{K}, \mathbb{A})$. 
Note that for $n=2$ this construction provides examples of global Veronesean caps. The Main Result of [9] states that these are the only global Veronesean caps, except for an example when $\mathbb{A}$ is the nonassociative but alternative Cayley-Dickson division algebra (but which we shall not need in the present paper since it has no analogue for local Veronesean caps that are not global Veronesean caps). Finally, the construction also works for $n=1$, and it gives us a parametrization of a standard $X$-ovoid of $\mathscr{V}_{n}(\mathbb{K}, \mathbb{A})$.

Now let $n \geq 3$. Given the pair $(\mathbb{K}, \mathbb{A})$ as above, and the standard local Veronesean cap $\mathscr{V}_{n}(\mathbb{K}, \mathbb{A})$ in the projective space $\mathbb{P}(V)$, then we call a subspace $S$ of $\mathbb{P}(V)$ admissible if $S$ does not meet the span of any pair of elliptic spaces. The projection of $\mathscr{V}_{n}(\mathbb{K}, \mathbb{A})$ from $S$ is also a local Veronesean cap, see Theorem 3.3 below, which we call a legal projection, and moreover proper if $S$ is nonempty.

Let $G$ be the little projective group of $\mathscr{P}^{n}(\mathbb{A})$, then we can define in the natural way the action of $G$ on any legal projection $X$ of $\mathscr{V}_{n}(\mathbb{K}, \mathbb{A})$. We say that $X$ is $G$-homogeneous if the stabilizer of $X$ in its ambient projective space induces that action of $G$ on $X$.

\subsection{Statement of the Main Results}

Main Result 1. Every finite-dimensional local Veronesean cap which is not a global Veronesean cap is projectively equivalent to a legal projection of $\mathscr{V}_{n}(\mathbb{K}, \mathbb{A})$, for some natural number $n \geq$ 3 , some commutative field $\mathbb{K}$ and some quadratic associative division algebra $\mathbb{A}$ over $\mathbb{K}$. In particular, the subdimension is a power of 2 , and if char $\mathbb{K} \neq 2$, then the subdimension can only take the values 1,2,4. Proper legal projections do not occur for $n=3$.

Some special cases of the Main Result are already known in the literature, such as the complete finite case. For a historic overview we refer to the introduction and to [9].

Main Result 2. Each G-homogeneous local Veronesean cap is projectively equivalent to a standard Veronesean cap.

The latter also includes the global Veronesean case.

\section{The projective space associated with a local Veronesean cap}

When dealing with a local Veronesean cap $(X, \Xi)$, it is tacitly assumed that $X$ spans the projective space $\mathbb{P}(V)$, for some vector space $V$ over a skew field $\mathbb{K}$. So let $\mathscr{V}=(X, \Xi)$ be a local Veronesean cap, where $X$ is a set of points in $\mathbb{P}(V)$, for some right vector space $V$ over the skew field $\mathbb{K}$, and $\Xi$ is a set of elliptic spaces satisfying (V1), (V2) and (V3) introduced before.

Associated with $\mathscr{V}$ we can consider the geometry $\mathscr{P}(X, \Xi)$ having point set $X$ and line set $\Xi$, endowed with the natural incidence. We usually write $\mathscr{P}$ for $\mathscr{P}(X, \Xi)$

Proposition 3.1 The geometry $\mathscr{P}$ is a (possibly infinite-dimensional) projective space. 
Proof If $\mathbb{K}$ is finite, then this follows from Lemma 3.1 of [12] and Theorem 3.1 of [13]. Henceforth we assume that $\mathbb{K}$ is an infinite skew field. In particular, every $X$-ovoid has infinitely many points (but the proof below works for $|\mathbb{K}| \geq 3$ ).

We use the characterization of (possibly infinite-dimensional) projective spaces given by Veblen \& Young [19]; for a modern account see Theorem 6.7.1 of [15]. By (V1) and the fact that every $X$-ovoid contains infinitely many points, we only have to show Veblen's axiom, i.e., if $O_{1}, O_{2}, O_{3}, O_{4}$ are four $X$-ovoids such that $O_{i} \cap O_{j}=\left\{x_{i j}\right\}$, for $1 \leq i<j \leq 4,(i, j) \neq(3,4)$, with $x_{12} \notin\left\{x_{13}, x_{14}, x_{23}, x_{24}\right\}$ and $O_{3} \neq O_{4}$, then $O_{3} \cap O_{4}$ is also a point of $X$.

We may assume that $O_{4}$ does not contain $x_{13}$ nor $x_{23}$. Put $W:=\left\langle O_{1}, O_{2}, O_{3}\right\rangle$ and $O_{5}:=$ $X\left(\left[x_{13}, x_{24}\right]\right)$. We claim that $O_{4}, O_{5} \subseteq W$. Indeed, since both $T_{x_{13}}\left(O_{3}\right)$ and $T_{x_{13}}\left(O_{1}\right)$ belong to $\left\langle O_{1}, O_{3}\right\rangle \subseteq W$, also $T_{x_{13}}\left(O_{5}\right)$ does by applying (V3) with as point $x_{13}$ and as $X$-ovoid $O_{2}$, and hence $O_{5}=\left\langle T_{x_{13}}\left(O_{5}\right), x_{24}\right\rangle \subseteq W$. Likewise, from applying (V3) to $x_{24}$ and $O_{1}$ we obtain $O_{4} \subseteq W$. Our claim is proved. Similar arguments show that $O_{1} \subseteq\left\langle O_{2}, O_{3}, O_{5}\right\rangle$, hence $W=\left\langle O_{2}, O_{3}, O_{5}\right\rangle$. We project $W \backslash\left\langle O_{2}\right\rangle$ from $\left\langle O_{2}\right\rangle$ onto a subspace $\Pi$ in $W$ complementary to $\left\langle O_{2}\right\rangle$ and denote the projection map by $\rho$. Set $x_{25}=x_{24}$ for notational reasons. Let $\Pi_{j}^{\infty}$ be the projection of $T_{x_{2 j}}\left(O_{j}\right)$, $j=3,4,5$.

Since each line in $\left\langle O_{j}\right\rangle$ through $x_{2 j}$ is either contained in $T_{x_{2 j}}\left(O_{j}\right)$ or intersects $O_{j}$ in a second point, the projection of $O_{j} \backslash\left\{x_{2 j}\right\}$ is an affine space in $\Pi$ which, completed with $\Pi_{j}^{\infty}$, becomes a projective space $\Pi_{j}, j=3,4,5$. Moreover, Axiom (V3) implies $\Pi_{4}^{\infty}=\Pi_{5}^{\infty}$.

We claim that $\rho$ is injective on $\left(W \backslash\left\langle O_{2}\right\rangle\right) \cap X$. Indeed, suppose two distinct points $y_{1}, y_{2} \in$ $\left(W \backslash\left\langle O_{2}\right\rangle\right) \cap X$ are such that $y_{1}^{\rho}=y_{2}^{\rho}$. Then $\left\langle y_{1}, y_{2}\right\rangle$ intersects $\left\langle O_{2}\right\rangle$ in some point $y$; by (V2) $y \in X\left(\left[y_{1}, y_{2}\right]\right) \cap O_{2}$, in particular $y \in X\left(\left[y_{1}, y_{2}\right]\right)$, contradicting the fact that $X\left(\left[y_{1}, y_{2}\right]\right)$ is a cap and $y_{1}, y_{2}, y$ are collinear. The claim is proved.

Since $W=\left\langle O_{2}, O_{3}, O_{5}\right\rangle$, we see that $\Pi=\left\langle\Pi_{3}, \Pi_{5}\right\rangle$. Notice that $x_{13}^{\rho} \in \Pi_{3} \cap \Pi_{5}$. We claim that $\left\{x_{13}^{\rho}\right\}=\Pi_{3} \cap \Pi_{5}$. Indeed, assume for a contradiction that some point $y \neq x_{13}^{\rho}$ is in $\Pi_{3} \cap \Pi_{5}$. Since there are infinitely many points on the line $\left\langle x_{13}^{\rho}, y\right\rangle$, we may assume that $y$ does not belong to either $\Pi_{3}^{\infty}$ or $\Pi_{5}^{\infty}$. Then there exist points $y_{k} \in O_{k} \backslash\left\{x_{13}\right\}, k=3,5$, with $y_{k}^{\rho}=y$, contradicting the injectivity of $\rho$ on $\left(W \backslash\left\langle O_{2}\right\rangle\right) \cap X$. The claim is proved.

Since $\left\{x_{13}^{\rho}\right\}=\Pi_{3} \cap \Pi_{5}$, it follows that $\Pi_{3}$ and $\Pi_{4}^{\infty}$ (as the latter equals $\Pi_{5}^{\infty}$ ) are complementary subspaces in $\Pi$. By symmetry, it follows that also $\Pi_{4}$ and $\Pi_{3}^{\infty}$ are complementary subspaces in $\Pi$. As a consequence, $\Pi_{3}$ and $\Pi_{4}$ intersect each other in a point $z$, and moreover, $z \notin \Pi_{3}^{\infty} \cup \Pi_{4}^{\infty}$. The injectivity of $\rho$ on $\left(W \backslash\left\langle O_{2}\right\rangle\right) \cap X$ then implies that the points of $O_{3}$ and $O_{4}$ which project onto $z$ coincide, and so $\mathrm{O}_{3}$ and $\mathrm{O}_{4}$ have a point in common, proving the proposition.

From now on we call the dimension of $\mathscr{P}$ the index of $(X, \Xi)$. If the index is equal to 2 , then all elliptic spaces intersect each other and hence $T(x, \xi)$ is independent of $\xi$, for every $x \in X$ and every $\xi \in \Xi$ with $x \notin \xi$. This means that Axiom (V3*) holds and that the local Veronesean cap is a global Veronesean cap. In [9] we showed the following theorem.

Theorem 3.2 Every global Veronesean cap, and hence every local Veronesean cap of index 2 , is projectively equivalent to $\mathscr{V}_{2}(\mathbb{K}, \mathbb{A})$, for some commutative field $\mathbb{K}$ and some quadratic alternative division algebra $\mathbb{A}$ over $\mathbb{K}$. In particular, the subdimension is either infinite or a power of 2 , and if char $\mathbb{K} \neq 2$, then the subdimension can only take the values $1,2,4,8$. 
We can now show one direction of our Main Result.

Theorem 3.3 Any legal projection of $\mathscr{V}_{n}(\mathbb{K}, \mathbb{A}), n \geq 3$, with $\mathbb{A}$ a quadratic associative division algebra over $\mathbb{K}$, is a local Veronesean cap with subdimension $\operatorname{dim}_{\mathbb{K}} \mathbb{A}$.

Proof First we show the assertion for $\mathscr{V}_{n}(\mathbb{K}, \mathbb{A}), n \geq 3$, itself.

We start by noting that, for every nonzero $a, b \in \mathbb{A}$, the mapping $\mathbb{A} \rightarrow \mathbb{A}: x \mapsto a x b$ is a linear permutation of the $\mathbb{K}$-vector space $\mathbb{A}$. It follows that $P L_{n+1}(\mathbb{A})$ (acting on the left) extends to $\mathbb{P}(V)$ in its natural action on $\mathscr{V}_{n}(\mathbb{K}, \mathbb{A})$. In particular, the transitivity of $\mathrm{PGL}_{n+1}(\mathbb{A})$ on the lines of $\mathscr{P}^{n}(\mathbb{A})$ implies that every line of $\mathscr{P}^{n}(\mathbb{A})$ is isomorphic to $\mathscr{V}_{1}(\mathbb{K}, \mathbb{A})$, the standard $X$-ovoid, and so (V1) holds. Now suppose $O_{1}$ and $O_{2}$ are two distinct $X$-ovoids. We distinguish two cases.

- Suppose $O_{1} \cap O_{2} \neq \emptyset$. Then, by the action of $\mathrm{PGL}_{n+1}(\mathbb{A})$, we may assume that $O_{1}$ consists of the points $\mathbb{K}\left(y_{i}, y_{(j, \ell)}\right)_{0 \leq i \leq n, 0 \leq j<\ell \leq n}$ of $\mathbb{P}(V)$ with only nonzero coordinates $y_{0}=x_{0} x_{0}^{\sigma}$, $y_{1}=x_{1} x_{1}^{\sigma}$ and $y_{0,1}=x_{0} x_{1}^{\sigma}, x_{0}, x_{1} \in \mathbb{A},\left(x_{0}, x_{1}\right) \neq(0,0)$, and similarly for $O_{2}$, where we have $y_{1}=x_{1} x_{1}^{\sigma}, y_{2}=x_{2} x_{2}^{\sigma}$ and $y_{1,2}=x_{1} x_{2}^{\sigma}, x_{1}, x_{2} \in \mathbb{A},\left(x_{1}, x_{2}\right) \neq(0,0)$. Then $\left\langle O_{1}\right\rangle \cap$ $\left\langle O_{2}\right\rangle=\{p\}$, where $p$ is the 1-space defined by a vector with all coordinates zero except for $y_{1}$, and the corresponding projective point belongs to $\mathscr{V}_{n}(\mathbb{K}, \mathbb{A})$.

- Suppose $O_{1} \cap O_{2}=\emptyset$. Then we may take $O_{1}$ as above, and $O_{2}$ can be redefined as consisting of the points $\mathbb{K}\left(y_{i}, y_{(j, \ell)}\right)_{0 \leq i \leq n, 0 \leq j<\ell \leq n}$ of $\mathbb{P}(V)$ with only nonzero coordinates $y_{2}=x_{2} x_{2}^{\sigma}, y_{3}=x_{3} x_{3}^{\sigma}$ and $y_{2,3}=x_{2} x_{3}^{\sigma}, x_{2}, x_{3} \in \mathbb{A},\left(x_{2}, x_{3}\right) \neq(0,0)$. So we have $\left\langle O_{1}\right\rangle \cap$ $\left\langle\mathrm{O}_{2}\right\rangle=\emptyset$.

This proves (V2). For (V3) we remark that the lines of $\mathscr{P}^{n}(\mathbb{A})$ through a fixed point and intersecting a given line not through that point all lie in a plane. By the action of $P G L_{n+1}(\mathbb{A})$ we may assume that this plane corresponds to $\mathscr{V} / 2(\mathbb{K}, \mathbb{A})$, where $(\mathrm{V} 3)$ holds by Theorem 3.3 of [9].

Now let $S$ be an admissible subspace of $\mathbb{P}(V)$ and let $X^{\prime}$ be the projection from $S$ of $\mathscr{V}_{n}(\mathbb{K}, \mathbb{A})$ onto some complementary subspace. Then, since $S$ does not contain any point of any elliptic space, all elliptic spaces are projected bijectively onto their image, so that (V1) holds for $X^{\prime}$. Also, since $S$ does not contain any point in the span of any two elliptic spaces, every pair of elliptic spaces is projected in an isomorphic way and so (V2) holds. Finally, since every point of the subspace generated by each global Veronesean subcap $\left(X_{1}, \Xi_{1}\right)$ (local of index 2) is contained in the span of two elliptic spaces of $\left(X_{1}, \Xi_{1}\right)$ (see Corollary 4.7 of [9]), no point of $S$ is contained in the subspace generated by any global Veronesean subcap (isomorphic to $\mathscr{V}_{2}(\mathbb{K}, \mathbb{A})$ ) of $\mathscr{V}_{n}(\mathbb{K}, \mathbb{A})$, and so (V3) holds for $X^{\prime}$.

\section{Local Veronesean caps of arbitrary finite index in projec- tive spaces of finite dimension}

Let $(X, \Xi)$ be a local Veronesean cap in the projective space $\mathbb{P}(V)$ (so $X$ spans $\mathbb{P}(V)$ ) and suppose $\operatorname{dim} V=N+1, N \in \mathbb{N}$. Suppose the index of $(X, \Xi)$ is equal to $n \in \mathbb{N}, n \geq 2$. By Theorem 3.2, 
we may suppose that $n \geq 3$. Let $\mathscr{P}$ be the associated projective space of dimension $n$. We will identify the points of $\mathscr{P}$ with the points of $X$. But when we want to emphasise the structure of $X$ as a projective space, we will mention $\mathscr{P}$, and when we want to emphasise the inclusion of $\mathscr{P}$ in $\mathbb{P}(V)$, then we mention $X$. Let $X_{1}$ be the set of points of a plane $\mathscr{P}_{1}$ of $\mathscr{P}$ and let $\Xi_{1}$ be the subset of $\Xi$ consisting of those elliptic spaces that contain at least two points of $X_{1}$. Then $\left(X_{1}, \Xi_{1}\right)$ is a global Veronesean cap in the subspace generated by $X_{1}$. Again by Theorem $3.2, \mathbb{K}$ is commutative and $\left(X_{1}, \Xi_{1}\right)$ is projectively equivalent to $\mathscr{V}_{2}(\mathbb{K}, \mathbb{A})$, for some quadratic alternative division ring $\mathbb{A}$ over $\mathbb{K}$. Since $n \geq 3$, we know that $\mathbb{A}$ is in fact associative. Also, since $N$ is finite, we know that $\operatorname{dim}_{\mathbb{K}} \mathbb{A}=: k$ is finite.

The proof of our Main Result has two parts. The strategy of the first part is based on [4]. With below terminology, the result is that we may assume that all hyperplanes of $\mathscr{P}$ are full. If $\mathbb{A}$ is not a purely inseparable extension of $\mathbb{K}$, and if char $\mathbb{K} \neq 2$ when $\mathbb{K}=\mathbb{A}$, then this implies that $N$ is maximal, and the Main Result follows, as we shall see. The second part is devoted to the case where $\mathbb{A}$ is a purely inseparable extension of $\mathbb{K}$, or $\mathbb{A}=\mathbb{K}$ and char $\mathbb{K}=2$. In that case, the fact that all hyperplanes of $\mathscr{P}$ are full does not imply that $N$ is maximal, and this complicates things significantly. When $\mathbb{A}=\mathbb{K}$, the arguments in [16] show a direct way to resolve this problem. However, when $\operatorname{dim}_{\mathbb{K}} \mathbb{A}>1$, then that method does not work well anymore. We use an entirely different method here which also works in the case $\mathbb{A}=\mathbb{K}$ and hence provides an alternative proof for the Main Result of [16].

The first part requires some new ideas compared to [4] since many arguments cease to hold in the infinite case. So we will be sketchy for the parts which do not need much adjusting from [4]. The second part is entirely new and will be explained in full detail.

We will need some basic properties of the standard Veronesean cap. We present those in Section 5 since it is a more natural place to prove them.

\subsection{First part of the proof}

First we note that, if $|\mathbb{K}|>2$, then $\Xi$ is determined by $X$.

\section{Lemma 4.1 If $|\mathbb{K}|>2$, then $X$ uniquely determines $\Xi$.}

Proof Let $O \subseteq X$ be an ovoid. We have to show that $O$ is contained in an elliptic space. Let $p_{1}, p_{2}$ be two arbitrary but fixed distinct points of $O$ and let $p_{3}$ be another arbitrary point. Since $|\mathbb{K}|>2$, there exists $p_{4} \in O \backslash\left\{p_{1}, p_{2}, p_{3}\right\}$ with $p_{1}, p_{2}, p_{3}, p_{4}$ coplanar. If $\left[p_{1}, p_{2}\right] \neq\left[p_{3}, p_{4}\right]$, then (V2) leads to the contradiction that the line through $p_{1}, p_{2}$ would contain at least three points of $X$. Hence $p_{3} \in\left[p_{1}, p_{2}\right]$ and the lemma follows.

For $x \in X$, let $T_{x}(X)$ be the subspace of $\mathbb{P}(V)$ generated by all $T_{x}(\xi)$, with $x \in \xi \in \Xi$. This notation is unambiguous if $|\mathbb{K}|>2$ by the previous lemma. If $|\mathbb{K}|=2$, then we let $\Xi$ be tacitly assumed. If no confusion is possible, we even abbreviate $T_{x}(X)$ to $T_{x}$.

Lemma 4.2 For all $x \in X$ we have that $T_{x}$ is the union of all $T_{x}(\xi)$ with $x \in \xi \in \Xi$, and also $\operatorname{dim} T_{x}=k n$. 
Proof This is proved by induction on $n$ in exactly the same way as Proposition 3.1 of [4], the result for $n=2$ being Fact 3 of [9]. Briefly, one chooses a hyperplane $Y$ in $\mathscr{P}$ with $x \in Y$ and a line $L$ of $\mathscr{P}$ with $x \in L \nsubseteq \subseteq Y$. Then $Y$ induces a local Veronesean cap in some subspace $H$ of $\mathbb{P}(V)$ and the induction hypothesis implies that $T_{x}(Y)$ is the union of all $T_{x}(\xi)$, with $\xi \in \Xi$ corresponding to a line of $Y$ through $x$. Moreover, $\operatorname{dim} T_{x}(Y)=k(n-1)$. Now let $\xi_{L} \in \Xi$ be the elliptic space corresponding to $L$, i.e, $X\left(\xi_{L}\right)=L$. Then $\operatorname{dim} T_{x}\left(\xi_{L}\right)=k$ and so $\operatorname{dim}\left\langle T_{x}(Y), T_{x}\left(\xi_{L}\right)\right\rangle=k n$. By considering planes of $\mathscr{P}$ through $L$, we see that every point of $\left\langle T_{x}(Y), T_{x}\left(\xi_{L}\right)\right\rangle$ is contained in some $T_{x}(\Pi)$, for $\Pi$ the point set of a plane of $\mathscr{P}$ containing $L$, and in fact $T_{x}(X)=\left\langle T_{x}(Y), T_{x}\left(\xi_{L}\right)\right\rangle$. Since the result holds true for $n=2$, the lemma then follows.

An immediate consequence is the following assertion (using (V2)).

Corollary 4.3 If $x \in X$ and $x \notin \xi \in \Xi$, then $T_{x} \cap \xi=\emptyset$.

Lemma 4.4 Let $Y$ be a hyperplane of $\mathscr{P}$ and let $x \in X \backslash Y$. Then $\left\langle Y, T_{x}\right\rangle=\langle X\rangle$.

Proof Consider $z \in X \backslash Y$. Then there exists $y \in Y$ with $[z, x] \cap Y=\{y\}$. Hence we obtain $z \in\left\langle y, T_{x}[x, z]\right\rangle \subseteq\left\langle Y, T_{x}\right\rangle$.

Since we have finite index and finite-dimensional ovoids we can bound the dimension of the ambient space $N$ of the local Veronesean cap by an easy induction argument, see Lemma 5.1 of [4]. More precisely, let $N(n, k)=\frac{1}{2} k n^{2}+\frac{1}{2} k n+n$.

Lemma 4.5 We always have $N \leq N(n, k)$. Also, if equality holds then for every hyperplane $Y$ in $\mathscr{P}$, we have $\operatorname{dim}\langle Y\rangle=N(n-1, k)$.

Proof For $n=2$ this is Theorem 3.2. For $n>2$ let $Y$ be a hyperplane, then by induction on $n$ and Lemmas 4.2 and 4.4, we have $N \leq \operatorname{dim}\langle Y\rangle+k n+1 \leq N(n-1, k)+k n+1=N(n, k)$. If equality holds, then the second inequality becomes an equality and implies $\operatorname{dim}\langle Y\rangle=N(n-$ $1, k)$.

One of the main steps is the following result, which is in the finite case Theorem 5.3 of [4]. Recall that $k=\operatorname{dim}_{\mathbb{K}} \mathbb{A}$, see the beginning of this section. The proof below is different from its finite analogue since we avoid the use of field automorphisms, which have an easier behaviour in the finite case.

Proposition 4.6 Let $(X, \Xi)$ be a local Veronesean cap of index $n$ and subdimension $k$ in $\mathbb{P}(V)$, with $\operatorname{dim} V=N(n, k)+1$. Then $X$ is projectively equivalent to the standard local Veronesean cap $\mathscr{V}_{n}(\mathbb{K}, \mathbb{A})$.

Proof We use induction on $n$, the result for $n=2$ being Theorem 3.2.

For $n>2$, let $\mathscr{V}_{n}(\mathbb{K}, \mathbb{A})$ be embedded in the projective space $\mathbb{P}(W)$, with $\operatorname{dim} W=N(n, k)+1$, and denote the point set of $\mathscr{V}_{n}(\mathbb{K}, \mathbb{A})$ by $\widetilde{X}$. Set $\mathscr{P}=\mathscr{P}(X, \Xi)$. Let $X_{1}$ and $X_{2}$ be the point sets of two distinct hyperplanes in $\mathscr{P}$, and let $L$ be the point set of a line of $\mathscr{P}$ not intersecting $X_{1} \cap X_{2}$. 
By Lemma 4.5 we have $\operatorname{dim}\left\langle X_{1}\right\rangle=\operatorname{dim}\left\langle X_{2}\right\rangle=N(n-1, k)$, and similarly $\operatorname{dim}\left\langle X_{1} \cap X_{2}\right\rangle=N(n-$ $2, k)$.

Now we claim that $\mathbb{P}(V)=\left\langle X_{1}, X_{2}, L\right\rangle$. Indeed, let $x \in X \backslash\left(X_{1} \cup X_{2} \cup L\right)$, then there is a unique plane $\pi$ of $\mathscr{P}$ containing $L$ and $x$, and $\pi$ also contains two lines $L_{1}, L_{2}$ in $X_{1}, X_{2}$, respectively. Hence, by Corollary 4.8 of [9], the corresponding global Veronesean cap is contained in $\left\langle L_{1}, L_{2}, L\right\rangle \subseteq\left\langle X_{1}, X_{2}, L\right\rangle$. This shows the claim. Now, this implies that $\operatorname{dim}\left(\left\langle X_{1}\right\rangle \cap\left\langle X_{2}\right\rangle\right) \leq$ $2 N(n-1, k)+k-N(n, k)=N(n-2, k)$, whereas $X_{1} \cap X_{2} \subseteq\left\langle X_{1}\right\rangle \cap\left\langle X_{2}\right\rangle$ leads to $\operatorname{dim}\left(\left\langle X_{1}\right\rangle \cap\right.$ $\left.\left\langle X_{2}\right\rangle\right) \geq N(n-2, k)$. Hence $\left\langle X_{1}\right\rangle \cap\left\langle X_{2}\right\rangle=\left\langle X_{1} \cap X_{2}\right\rangle$.

Let, for clarity, $(\widetilde{X}, \widetilde{\Xi})$ be the standard local Veronesean cap $\mathscr{V}_{n}(\mathbb{K}, \mathbb{A})$. By the induction hypothesis, $X_{2}$ is projectively isomorphic to the standard local Veronesean cap $\mathscr{V}_{n-1}(\mathbb{K}, \mathbb{A})$, which can be obtained from $(\widetilde{X}, \widetilde{\Xi})=\mathscr{V}_{n}(\mathbb{K}, \mathbb{A})$ by intersecting with an appropriate $N(n-1, k)$-space. Let $\widetilde{X}_{2}$ be that intersection; then $\left\langle\widetilde{X}_{2}\right\rangle$ is the mentioned appropriate $N(n-1, k)$-space. Also, we have an isomorphism $\varphi:\left\langle X_{2}\right\rangle \rightarrow\left\langle\widetilde{X}_{2}\right\rangle$ mapping $X_{2}$ to $\widetilde{X}_{2}$. It follows directly from the well-known Fundamental Theorem of Projective Geometry (see e.g. Chapter III of [2]) that there exists an isomorphism $\mathscr{P}(X, \Xi) \rightarrow(\mathscr{P}(\widetilde{X}, \widetilde{\Xi}))$ inducing a map $\theta: X \rightarrow \widetilde{X}$ such that the restriction of $\theta$ to $X_{2}$ coincides with $\varphi$ on $X_{2}$. Let $X_{1}^{\theta}=\widetilde{X}_{1}, L^{\theta}=\widetilde{L}$ and, selecting $c \in X \backslash\left(X_{1} \cup X_{2} \cup L\right)$, let $c^{\theta}=\widetilde{c}$. By Lemmas 4.2 and 4.4, $T_{c}$ is a subspace of $\mathbb{P}(V)$ complementary to both $\left\langle X_{1}\right\rangle$ and $\left\langle X_{2}\right\rangle$. Since each member $\xi$ of $\Xi$ through $c$ intersects $T_{c}$ in a hyperplane of $\xi$, the projection $\rho$ of $\left\langle X_{1}\right\rangle$ from $T_{c}$ onto $\left\langle X_{2}\right\rangle$ induces a bijection of $X_{1}$ onto $X_{2}$, which coincides in $\mathscr{P}$ with the projection of $X_{1}$ to $X_{2}$ from $c$. Since the latter projection is independent of the local Veronesean cap $(X, \Xi)$, the projection $\widetilde{\rho}$ from $T_{\widetilde{c}}$ mapping $\left\langle\widetilde{X}_{1}\right\rangle$ onto $\left\langle\widetilde{X}_{2}\right\rangle$ maps $\widetilde{X}_{1}$ onto $\widetilde{X}_{2}$. It follows that $\rho \varphi \widetilde{\rho}^{-1}$ is an isomorphism from $\left\langle X_{1}\right\rangle$ to $\left\langle\widetilde{X}_{1}\right\rangle$ mapping $X_{1}$ bijectively onto $\widetilde{X}_{1}$, and coinciding with $\varphi$ over $X_{1} \cap X_{2}$. Hence we can extend $\varphi$ to $\left\langle X_{1}\right\rangle \cup\left\langle X_{2}\right\rangle$, and subsequently to $\left\langle X_{1}, X_{2}\right\rangle$; the latter is mapped projectively onto $\left\langle\widetilde{X}_{1}, \widetilde{X}_{2}\right\rangle$. Moreover, the restriction of $\varphi$ to $X_{1} \cup X_{2}$ coincides with $\theta$ restricted to $X_{1} \cup X_{2}$.

Let $x \in X_{1} \cap X_{2}$ be arbitrary but such that the plane $\Pi$ of $\mathscr{P}$ containing $x$ and $L$ does not contain c. Put $\Pi^{\theta}=\widetilde{\Pi}$. Similarly as above, we use the projection from $c$ of $\Pi$ onto $X_{2}$ to obtain that we can extend $\varphi$ to $\left\langle X_{1}, X_{2}, L\right\rangle$ and that the restriction of $\varphi$ to $X_{1} \cup X_{2} \cup \Pi$ coincides with $\theta$ restricted to $X_{1} \cup X_{2} \cup \Pi$.

Now, since $\left\langle X_{1}, X_{2}, L\right\rangle$ is the whole point set of $\mathbb{P}(V)$, we are only left to show that $\varphi$ coincides with $\theta$ over $X$. This is already true over $X_{1} \cup X_{2} \cup \Pi$. Let $p$ be any point of $X \backslash\left(X_{1} \cup X_{2} \cup \Pi\right)$. Then the plane $\Pi_{p}$ of $\mathscr{P}$ generated by $p$ and $L$ intersects $X_{1} \cap X_{2}$ in a point $x_{0}$; let $x_{1}=L \cap X_{1}$ and $x_{2}=L \cap X_{2}$. Put $x_{j}^{\theta}=\tilde{x}_{j}, j=0,1,2$. Since $T_{x_{0}}$ is determined by $X_{1} \cup X_{2}$ and $T_{x_{j}}$ is determined by $X_{j} \cup L, j=1,2$, and likewise for $T_{\widetilde{x}_{j}}, j=0,1,2$, we see that $\varphi$ maps $T_{x_{j}}$ onto $T_{\widetilde{x}_{j}}, j=0,1,2$.

Now $\Pi_{p}$ and $\widetilde{\Pi}_{p}=\Pi_{p}^{\theta}$ are local Veronesean caps of index 2 and the previous paragraph implies that $\varphi$ maps the tangent spaces $T_{x_{j}}\left(\Pi_{p}\right)$ (with obvious notation) to the tangent spaces $T_{\widetilde{x}_{j}}\left(\widetilde{\Pi}_{p}\right)$, $j=0,1,2$. Now put $x_{j j^{\prime}}=\left\langle p, x_{j^{\prime \prime}}\right\rangle \cap\left\langle x_{j}, x_{j^{\prime}}\right\rangle,\left\{j, j^{\prime}, j^{\prime \prime}\right\}=\{0,1,2\}$. Then, by Lemma 6.4 of [9], we have $\{p\}=\left\langle T_{x_{0}}\left(\Pi_{p}\right), x_{12}\right\rangle \cap\left\langle T_{x_{1}}\left(\Pi_{p}\right), x_{02}\right\rangle \cap\left\langle T_{x_{2}}\left(\Pi_{p}\right), x_{01}\right\rangle$, and likewise $\left\{p^{\theta}\right\}=$ $\left\langle T_{\widetilde{x}_{0}}\left(\widetilde{\Pi}_{p}\right), x_{12}^{\varphi}\right\rangle \cap\left\langle T_{\widetilde{x}_{1}}\left(\widetilde{\Pi}_{p}\right), x_{02}^{\varphi}\right\rangle \cap\left\langle T_{\widetilde{x}_{2}}\left(\widetilde{\Pi}_{p}\right), x_{01}^{\varphi}\right\rangle$. This implies that $p^{\varphi}=p^{\theta}$ and the proposition is proved.

Subspaces $Y$ of $\mathscr{P}$ with $X \cap\langle Y\rangle=Y$ will be called full. The analogue in the finite case of the following lemma is Lemma 5.4 of [4], but its proof is not valid in the present infinite case 
(mainly because of the existence of the case of an inseparable field extension). Hence we present another proof.

Lemma 4.7 Let $\pi \subseteq X$ be a plane of $\mathscr{P}$. Then $\pi$ is full.

Proof Suppose to the contrary that $p$ is a point of $X \backslash \pi$ in $\langle\pi\rangle$. By Corollary 4.7 of [9], there are two distinct elliptic spaces $\xi_{1}$ and $\xi_{2}$ corresponding to two respective lines of $\pi$ such that $p \in\left\langle\xi_{1}, \xi_{2}\right\rangle$. Set $x=\xi_{1} \cap \xi_{2}$. If $p$ would be in $T_{x}(\pi)$, then it would be contained in some elliptic space $\xi \subseteq \pi$ by Lemma 4.2, which is a contradiction. Hence the unique plane $\alpha$ of $\mathbb{P}(V)$ containing $p$ and $x$ and intersecting both $\xi_{1}$ and $\xi_{2}$ in a respective line $L_{1}$ and $L_{2}$ has the property that at least one of $L_{1}$ or $L_{2}$, say $L_{1}$, is not tangent to $X \cap \xi_{1}$. Hence $L_{1}$ contains a point $y_{1} \in \pi \backslash\{x\}$. But then $\left[p, y_{1}\right]$ intersects $\xi_{2}$ in a point $y_{2} \in\left\langle p, y_{1}\right\rangle$ of $L_{2}$ distinct from $x$, contradicting the fact that $X$ is a cap.

The proof of the following lemma is again similar to the finite case, Theorem 5.5 of [4].

Lemma 4.8 Let $(X, \Xi)$ have index 3. Then $N=N(3, k)=6 k+3$.

Proof By Lemma 4.5, $N \leq N(3, k)$. Suppose now for a contradiction that $N<N(3, k)$. Let $\pi$ be any (hyper)plane in $\mathscr{P}$. Then $\operatorname{dim}\langle\pi\rangle=3 k+2$. Lemma 4.2 implies that, for any $x \in X \backslash \pi$, we have $\operatorname{dim} T_{x}=3 k$. Consequently $T_{x} \cap\langle\pi\rangle \neq \emptyset$. Let $a \in T_{x} \cap\langle\pi\rangle$, then by Lemma 4.2 there exists $\xi \in \Xi$ such that $a \in T_{x}(\xi)$. Set $y:=X(\xi) \cap \pi$. As the line $\langle a, y\rangle$ belongs to $\xi$ and $\xi \cap \pi=\{y\}$ by Lemma 4.7, we have $a \in T_{y}(\xi) \subseteq T_{y}$. Now $a \notin T_{y}(\pi)$ as otherwise, by Lemma 4.2, $a \in \xi^{\prime} \in \Xi$ with $X\left(\xi^{\prime}\right) \subseteq \pi$, and so $a \in \xi \cap \xi^{\prime}$, contradicting (V2). Hence $\operatorname{dim}\left(T_{y} \cap\langle\pi\rangle\right)>\operatorname{dim} T_{y}(\pi)=2 k$. Now let $\xi^{\prime \prime} \in \Xi$ with $y \notin \xi^{\prime \prime}$ and $X\left(\xi^{\prime \prime}\right) \subseteq \pi$. Then by a dimension argument $T_{y} \cap \xi^{\prime \prime} \neq \emptyset$, contradicting Corollary 4.3.

Note that Lemma 4.1 of [16] leaves the possibility $N(3,1)=8$ open. With the above lemma, this is now excluded and then Proposition 4.2 of [16] becomes superfluous.

The analogue of Lemma 5.6 of [4] would be that, if $N<N(n, k)$, then some hyperplane of $\mathscr{P}$ is not full. However, this is only true in the case where either $\mathbb{A}$ is non-abelian, or $\mathbb{A}$ is a separable field extension of $\mathbb{K}$, or $\mathbb{K}=\mathbb{A}$ and char $\mathbb{K} \neq 2$, as we will show now. We shorten an argument in the proof of Lemma 5.6 of [4].

Lemma 4.9 Let $(X, \Xi)$ be a local Veronesean cap with index $n$ and subdimension $k$. If $N<$ $N(n, k)$, and if $\mathbb{A}$ is not an inseparable extension of $\mathbb{K}$, and char $\mathbb{K} \neq 2$ if $\mathbb{A}=\mathbb{K}$, then there exists a non-full hyperplane of $\mathscr{P}$.

Proof Note that the assumptions on $\mathbb{A}$ are equivalent to saying that the $X$-ovoids have a trivial nucleus, i.e., if $Q$ is an $X$-ovoid, then no point of $\langle Q\rangle$ is contained in every tangent hyperplane of $Q$.

We prove this by induction on the index $n$, the case $n=2$ is Theorem 3.2 and the case $n=3$ is Lemma 4.8 (in these cases $N$ is always equal to $N(n, k)$ ). So suppose $n \geq 4$.

Let $Y \subseteq X$ be a hyperplane of $\mathscr{P}$. If it is not full, we are done. So suppose it is and first suppose that $\operatorname{dim}\langle Y\rangle<N(n-1, k)$. Then by induction there is a non-full hyperplane of $Y$, say $Z$. Then the hyperplane spanned by $Z$ and a point $x \in X \backslash Y$ is not full. 
So suppose that $\operatorname{dim}\langle Y\rangle=N(n-1, k)$. Then by a dimension argument, for all $x \in X \backslash Y$, there exists $a \in T_{x} \cap\langle Y\rangle$. By Lemma 4.2, there exists a point $y \in Y$ with $a \in T_{x}([x, y])$. Exactly as in the proof of the previous lemma, we obtain $\operatorname{dim}\left(T_{y} \cap\langle Y\rangle\right)>k(n-1)$.

Pick a hyperplane $Z$ of the subspace $Y$ of $\mathscr{P}$ not containing $y$, then, by Lemma 4.5, we have $\operatorname{dim}\langle Z\rangle=N(n-2, k)$. Hence by the above there exists a point $b \in T_{y} \cap\langle Z\rangle$, and since $T_{y}(Y) \cap$ $\langle Z\rangle=\emptyset$, there exists a $w \in X \backslash Y$ with $b \in\langle[y, w]\rangle$ and we have $[y, w] \cap Y=y \notin Z$. By Lemma $4.6, Z$ is projectively equivalent to the standard local Veronesean cap $\mathscr{V}_{n-2}(\mathbb{K}, \mathbb{A})$. Hence, since $X([y, w])$ has a trivial nucleus, there exist two points $x_{1}, x_{2} \in[y, w]$ with $b \in\left\langle x_{1}, x_{2}\right\rangle$. Since $y \notin Z$ and $w \notin Y$, we have $Z \cap[w, y]=\emptyset$. Hence, in $\mathscr{P}$, the subspace $Z$ and the point $x_{1} \in[w, y]$ generate a hyperplane $Y^{\prime}$, which does not contain $x_{2}$. But $x_{2} \in\left\langle Z, x_{1}\right\rangle \subseteq\left\langle Y^{\prime}\right\rangle$. So $Y^{\prime}$ is not full.

In case a non-full hyperplane exists, we will show, using the technique of Section 6 of [4], that $X$ is a quotient (or, in geometric terms, a projection) of a local Veronesean cap in a higherdimensional space. The finite-dimensionality of the projective space then implies that $X$ is a quotient of a local Veronesean cap without non-full hyperplanes. Then, in the case that $X$ ovoids have trivial nuclei, by Lemma $4.9, N=N(n, k)$ and Lemma 4.6 completes the proof of Main Result 1 in that case.

In order to show that an object $F$ is the central projection of another object $F^{\prime}$ from a point, one may always choose $F^{\prime}$ in such a way that $F^{\prime} \cap\langle F\rangle$ is a pre-assigned hyperplane section $H$ of $F^{\prime}$, and candidates for that are, in our case, the hyperplane sections of the standard local Veronesean cap. Moreover, it is convenient that $H$ spans $\langle F\rangle$, as this shows that $\langle F\rangle$ is a hyperplane of $\left\langle F^{\prime}\right\rangle$ and thus the projection $F^{\prime} \rightarrow F$ is not the restriction of an isomorphism $\left\langle F^{\prime}\right\rangle \rightarrow\langle F\rangle$ of projective spaces. The points of $H$ are then fixed under the projection. In our case, the simplest geometric hyperplanes to handle are the Hermitian pencils of hyperplanes, which we introduce now.

Lemma 4.10 Let the standard local Veronesean cap $\mathscr{V}_{n}(\mathbb{K}, \mathbb{A})=(X, \Xi)$ in the projective space $\mathbb{P}$ correspond to the projective space $\mathscr{P}$. Let $Z \subseteq \mathscr{V}_{n}(\mathbb{K}, \mathbb{A})$ be an $(n-2)$-dimensional subspace of $\mathscr{P}$ and let the $X$-ovoid $Q \subseteq \mathscr{V}_{n}(\mathbb{K}, \mathbb{A})$ correspond to a line of $\mathscr{P}$ disjoint from $Z$. Let $Y_{0}$ and $Y_{1}$ be two distinct hyperplanes of $\mathscr{P}$ both containing $Z$. Let $H$ be a hyperplane of $\langle Q\rangle$ containing $\left(Y_{0} \cup Y_{1}\right) \cap Q$. For a point $x \in H \cap Q$, let $Y_{x} \subseteq \mathscr{V}_{n}(\mathbb{K}, \mathbb{A})$ be the hyperplane in $\mathscr{P}$ containing $Z$ and $x$. Then the union $\mathscr{U}(Z, Q \cap H)$ of all $Y_{x}$, with $x$ ranging over $H \cap Q$, spans a hyperplane of $\mathbb{P}$.

Proof Put $\mathscr{U}=\mathscr{U}(Z, Q \cap H)$ for short. Let $X$ be the point set of $\mathscr{V}_{n}(\mathbb{K}, \mathbb{A})$. Put $x_{j}=Y_{j} \cap Q$, $j=0,1$. We project $X \backslash Y_{0}$ from $\left\langle Y_{0}\right\rangle$ onto $T_{x_{1}}$. By Proposition 5.7, this projection is injective, and the $Y_{x}, x \in(H \cap Q) \backslash\left\{x_{0}\right\}$ project into affine spaces of dimension $k(n-1)$ which all share the same projective completion (a projective space $W$ of dimension $k n-k-1$ ). All these affine spaces intersect the projection of $H \backslash\left\langle Y_{0}\right\rangle$, which is a hyperplane $H^{\prime}$ of the projection $L^{\prime}$ (a $k$-dimensional affine space, see Proposition 5.7) of $\langle Q\rangle \backslash\left\langle Y_{0}\right\rangle$. Since $Q \cap Z=\emptyset$, Remark 5.8 implies that $W \cap\left\langle L^{\prime}\right\rangle=\emptyset$. A dimension argument now shows that $T_{x_{1}}=\left\langle W, L^{\prime}\right\rangle$. But the projection of $\mathscr{U} \backslash Y_{0}$ coincides with $\left\langle W, H^{\prime}\right\rangle \backslash W$. Since $\left\langle W, H^{\prime}\right\rangle$ is a hyperplane of $\left\langle W, L^{\prime}\right\rangle$, the lemma follows.

The set of points of $\mathscr{P}$ corresponding to $\mathscr{U}(Z, Q \cap H)$ in the statement of the previous lemma will be called a Hermitian pencil of hyperplanes (of $\mathscr{P}$ ). Note that, although we defined this 
notion in the standard Veronesean cap, it also lives, as a subset of the point set $X$, in the local Veronesean cap $(X, \Xi)$ through the identification of $X$ with the point set of $\mathscr{P}$.

Lemma 4.11 Let $(X, \Xi)$ be a local Veronesean cap in $\mathbb{P}(V)$ with index $n$ and subdimension $k$. If $Y_{0} \subseteq X$ is a non-full hyperplane of $\mathscr{P}$ with $x \in\left(X \cap\left\langle Y_{0}\right\rangle\right) \backslash Y_{0}$, then $X$ is the image of a local Veronesean cap $X^{\prime}$ (with corresponding projective geometry $\mathscr{P}^{\prime}$ isomorphic to $\mathscr{P}$ ) under a central projection $\rho$ such that for the unique point $x^{\prime} \in X^{\prime}$ with $x^{\prime \rho}=x$ and the unique hyperplane $Y_{0}^{\prime} \subseteq X^{\prime}$ of $\mathscr{P}^{\prime}$ with $Y_{0}^{\prime \rho}=Y_{0}$ we have $x^{\prime} \notin\left\langle Y_{0}^{\prime}\right\rangle$.

Proof First note that, since every plane of $\mathscr{P}$ induces the structure of a global Veronesean cap on $X$, and also on $\mathscr{V}_{n}(\mathbb{K}, \mathbb{A})$, Theorem 3.2 implies that, if $Q$ is any $X$-ovoid, and if $Q^{\prime}$ is the corresponding quadric in $\mathscr{V}_{n}(\mathbb{K}, \mathbb{A})$, then hyperplanes in $\langle Q\rangle$ intersecting $Q$ nontrivially correspond bijectively to hyperplanes in $\left\langle Q^{\prime}\right\rangle$ intersecting $Q^{\prime}$ nontrivially.

Now, by Lemma 4.8, we have $n>3$. Let $Z$ be an $(n-2)$-space of $\mathscr{P}$ contained in $Y_{0}$ and let $Q$ be an $X$-ovoid disjoint from $Z$ and containing $x$. Consider a hyperplane $H$ of the subspace $\langle Q\rangle$ which does not contain $x$, but which does contain at least two points of $Q$ among which $Y_{0} \cap Q$. Let $\mathscr{U}=\mathscr{U}(Z, Q \cap H)$ be the corresponding Hermitian pencil of hyperplanes (well-defined by the previous paragraph).

We claim that $\langle\mathscr{U}\rangle=\mathbb{P}(V)$. Indeed, let $u$ be any point of $X$, and we may assume that $u \notin \mathscr{U}$. If we show that $u \in\langle\mathscr{U}\rangle$, then we have $\mathbb{P}(V)=\langle X\rangle \subseteq\langle\mathscr{U}\rangle \subseteq \mathbb{P}(V)$ and $\langle\mathscr{U}\rangle=\mathbb{P}(V)$ follows.

If $u \in Q$, then there is nothing to prove since $u \in\langle Q \cap H, x\rangle=\langle Q\rangle$. (This also shows $Q \subseteq\langle\mathscr{U}\rangle$.) Hence we may assume that $u$ and $Q$ are contained in a unique plane $\pi$ of $\mathscr{P}$, which intersects $Z$ in a unique point $z$. The plane $\pi$ induces a global Veronesean cap of index 2 on $X$. Let $Y$ be any hyperplane of $\mathscr{P}$ through $Z$ inside $\mathscr{U}$ different from $Y_{0}$. Then $\pi \cap Y$ is a quadric that corresponds to a line in $\pi$, and it follows that $\pi \cap \mathscr{U}$ is a Hermitian pencil of lines in $\pi$. Hence, by Lemma 4.10, $\langle\pi \cap \mathscr{U}\rangle$ is a hyperplane of $\langle\pi\rangle$. But $x \in\langle\pi\rangle \backslash \mathscr{U}$ and $x \in\langle\mathscr{U}\rangle$. It follows that $\langle\pi\rangle \subseteq\langle\mathscr{U}\rangle$ and hence $u \in\langle\mathscr{U}\rangle$. The claim is proved.

Now we embed $\mathbb{P}(V)$ as a hyperplane in $\mathbb{P}\left(V^{\prime}\right)$, where $V^{\prime}$ is a vector space of dimension $N+$ 2 over $\mathbb{K}$ containing $V$ as an $(N+1)$-dimensional subspace. Let $c$ be an arbitrary point in $\mathbb{P}\left(V^{\prime}\right) \backslash \mathbb{P}(V)$. We want to define for each point $p \in X$ a point $p^{\theta} \in \mathbb{P}\left(V^{\prime}\right)$ such that $p, p^{\theta}, c$ are collinear and $X^{\theta}=\left\{y^{\theta}: y \in X\right\}$ is the point set of a local Veronesean cap in $\mathbb{P}\left(V^{\prime}\right)$ with index $n$ and subdimension $k$ isomorphic to $(X, \Xi)$ (and the restriction to $X^{\theta}$ of the natural projection of $\mathbb{P}\left(V^{\prime}\right) \backslash\{c\}$ from $c$ onto $\mathbb{P}(V)$ is an isomorphism). We pick $x^{\theta} \neq c$ in $\mathbb{P}\left(V^{\prime}\right) \backslash \mathbb{P}(V)$ such that $c, x$ and $x^{\theta}$ are collinear.

For $y \in \mathscr{U}$ we set $y^{\theta}:=y$. Since $\mathscr{U}$ spans $\mathbb{P}(V)$, we will certainly have that $X^{\theta}$ will span $\mathbb{P}\left(V^{\prime}\right)$, as the latter is spanned by $\mathbb{P}(V)$ and $x^{\theta}$.

For $y \in X \backslash \mathscr{U}$, consider the set $C_{y}:=\mathscr{U} \cap[x, y]$. If $X([x, y]) \cap Z=\emptyset$, then $C_{y}$ contains at least two points. If $X([x, y]) \cap Z \neq \emptyset$, then $C_{y}$ contains a unique point, say $t \in Z$. Either way, since on the standard local Veronesean cap $\mathscr{V}_{n}(\mathbb{K}, \mathbb{A})$, the set $\mathscr{U}$ corresponds to a hyperplane of the ambient projective space, and $y$ to a point not contained in there, the set $C_{y}$ is a non-empty hyperplane section of $[x, y]$, say $C_{y}=H_{y} \cap X([x, y])$, with $H_{y}$ a hyperplane in $[x, y]$. We define $y^{\theta}:=\langle c, y\rangle \cap$ $\left\langle H_{y}, x^{\theta}\right\rangle$. Note that these indeed have a unique point in common, as we are considering the intersection of a $k$-space and a line (which are not incident) inside the $(k+1)$-space $\left\langle[x, y], x^{\theta}\right\rangle$. 
Finally, we define $X^{\theta}:=\left\{y^{\theta}: y \in X\right\}, L^{\theta}:=\left\{y^{\theta} \mid y \in L\right\}$ for $L \in \mathscr{L}$, and $\Xi^{\theta}:=\left\{\left\langle L^{\theta}\right\rangle \mid L \in \mathscr{L}\right\}$. We now show that $\left(X^{\theta}, \Xi^{\theta}\right)$ is a local Veronesean cap of index $n$ and subdimension $k$ isomorphic to $(X, \Xi)$. To that aim, let $S \subseteq X$ correspond to the point set of a 3 -space of $\mathscr{P}$, with $x \in S$ and such that $S \cap Z$ is a line of $\mathscr{P}$. Then $\mathscr{U} \cap S$ is a Hermitian pencil of hyperplanes in $S$. Lemmas 4.8, 4.10 and the definition of Hermitian pencil of hyperplanes assert that $\mathscr{U} \cap S$ spans a hyperplane of $\langle S\rangle$. By the definition of $\theta$, whenever $y \in S \backslash \mathscr{U}$, we have $y^{\theta} \in\left\langle\mathscr{U} \cap S, x^{\theta}\right\rangle \backslash\langle\mathscr{U} \cap$ $S\rangle$. Hence, for an arbitrary such point $y$, we also have $x^{\theta} \in\left\langle\mathscr{U} \cap S, y^{\theta}\right\rangle$. Moreover, $\left\langle\mathscr{U} \cap S, x^{\theta}\right\rangle$ is a hyperplane of $\langle S, c\rangle$ not containing $c$ (otherwise it would also contain $x$ and hence $\langle S\rangle$, a contradiction). It follows that $\theta$ restricted to $S$ is the projection of $\langle S\rangle$ onto $\left\langle\mathscr{U} \cap S, x^{\theta}\right\rangle$ from $c$ restricted to $S$. This now has two important consequences:

(1) $S^{\theta}=\left\{y^{\theta}: y \in S\right\}$ is isomorphic to the standard local Veronesean cap $\mathscr{V} / 3(\mathbb{K}, \mathbb{A})$;

(2) the definition of $\theta$ does not depend on the pair $\left(x, x^{\theta}\right)$. Indeed, let $y, z \in X \backslash \mathscr{U}$ be arbitrary and suppose we define $z^{\theta}$ starting from the pair $\left(y, y^{\theta}\right)$. We can include the triplet of points $x, y, z \in X \backslash \mathscr{U}$ in a 3 -space of $\mathscr{P}$ intersecting $Z$ in a line. Then the above arguments show that $U_{S}=\left\langle\mathscr{U} \cap S, x^{\theta}\right\rangle=\left\langle\mathscr{U} \cap S, y^{\theta}\right\rangle$ is independent of $x$, and so is $z^{\theta}=\langle c, z\rangle \cap U_{S}$.

We now check the properties (V1), (V2), (V3).

V1 Every pair of points of $X^{\theta}$ is contained in a quadric of dimension $k$ since we can include every pair of points of $\mathscr{P}$ in a 3 -space $S$ of $\mathscr{P}$ intersecting $Z$ in a line, and then the quadric we are looking for belongs to $S^{\theta}$. This is (V1).

V2 Let $L_{1}, L_{2}$ be two arbitrary lines of $\mathscr{P}$. Suppose that they generate a subspace $W$ of $\mathscr{P}$ that contains a plane of $Z$. One possibility is that they are both contained in $Z$. Since $Z^{\theta}=Z$, it follows that $\left\langle L_{1}^{\theta}\right\rangle \cap\left\langle L_{2}^{\theta}\right\rangle=L_{1}^{\theta} \cap L_{2}^{\theta}$. The other possibility is that they are not contained in $Z$, but are contained in a hyperplane $Y$ of $\mathscr{P}$ that contains $Z$. If $Y \subseteq \mathscr{U}$, then again $L_{1}^{\theta}=L_{1}$ and $L_{2}^{\theta}=L_{2}$ and $\left\langle L_{1}^{\theta}\right\rangle \cap\left\langle L_{2}^{\theta}\right\rangle=L_{1}^{\theta} \cap L_{2}^{\theta}$. If $Y$ is not contained in $\mathscr{U}$, then $W \cap \mathscr{U}=W \cap Z$. Let $T$ be the unique hyperplane of $\langle W\rangle$ intersecting $W$ in $W \cap Z$. It follows from the definition of $\theta$ and the independence of $x$ noted above that for any point $y \in W \backslash Z$ we have $\left\langle W^{\theta}\right\rangle=\left\langle T, y^{\theta}\right\rangle$, and again $W^{\theta}$ is the bijective projection from $c$ of $W$. So again $\left\langle L_{1}^{\theta}\right\rangle \cap\left\langle L_{2}^{\theta}\right\rangle=L_{1}^{\theta} \cap L_{2}^{\theta}$.

If $W$ does not contain a plane of $Z$, then we can include it in a 3 -space of $\mathscr{P}$ intersecting $Z$ in a line of $\mathscr{P}$, and then $\left\langle L_{1}^{\theta}\right\rangle \cap\left\langle L_{2}^{\theta}\right\rangle=L_{1}^{\theta} \cap L_{2}^{\theta}$ follows from the projection property from $c$. We have shown that (V2) holds.

V3 Finally, we must show that (V3) holds. In fact, this is true whenever any point and line in $\mathscr{P}$ can be included in a global Veronesean subcap in $X^{\theta}$. But this follows from the fact that any plane of $\mathscr{P}$ either is contained in $Z$, or can be included in a 3-space of $\mathscr{P}$ intersecting $Z$ in a line.

We have shown that $(X, \Xi)$, where $X$ spans an $N$-space, is the image of $\left(X^{\theta}, \Xi^{\theta}\right)$, where $X^{\theta}$ spans an $(N+1)$-space, under a central projection. Hence, putting $X^{\theta}=X^{\prime}$ and $\rho$ the central projection of $X^{\prime}$ onto $X$ from $c$, concludes the proof. 
So starting from a non-full hyperplane of $\mathscr{P}$, we can apply the "lifting"-construction of the previous lemma. If we find another non-full hyperplane, then we can repeat this construction over and over again until all hyperplanes are full. That this procedure ends after a finite number of steps is guaranteed by the fact that, if the dimension of the surrounding projective space is $N(n, k)$, then all hyperplanes are full.

Hence we can state the following proposition.

Proposition 4.12 Every local Veronesean cap $(X, \Xi)$ (with corresponding projective space $\mathscr{P}$ ) in the projective space $\mathbb{P}(V)$ with index $n$ and subdimension $k$ is the (bijective) projection of a local Veronesean cap $\left(X^{\prime}, \Xi^{\prime}\right)$ in some projective space $\mathbb{P}\left(V^{\prime}\right)$ containing $\mathbb{P}(V)$, where all hyperplanes of $\mathscr{P}$ are full in $\left(X^{\prime}, \Xi^{\prime}\right)$. In particular, if $\mathbb{A}$ is not an inseparable extension of $\mathbb{K}$ and char $\mathbb{K} \neq 2$ if $\mathbb{A}=\mathbb{K}$, then $\left(X^{\prime}, \Xi^{\prime}\right)$ is isomorphic to the standard local Veronesean cap $\mathscr{V}_{n}(\mathbb{K}, \mathbb{A})$ and $(X, \Xi)$ is a legal projection thereof.

Proof The first assertion follows from the paragraph preceding the proposition. The second assertion follows from Lemma 4.9 and Proposition 4.6.

\subsection{Second part of the proof}

Hence, in order to have a complete proof of Main Result 1, we may, in view of Proposition 4.12, from now make the following

\section{Assumptions}

- every hyperplane of $\mathscr{P}$ is full

- the characteristic of $\mathbb{K}$ is $2, \mathbb{A}=\mathbb{K}$ or $\mathbb{A}$ is a purely inseparable extension of $\mathbb{K}$ such that $\mathbb{K}$ contains the squares of all elements of $\mathbb{A}$.

- In view of Proposition 4.6, we may still assume that $N<N(n, k)$.

Lemma 4.13 Under the above assumptions, there exists a hyperplane $Y$ of $\mathscr{P}$ and a point $x \in X \backslash Y$ such that $T_{x} \cap\langle Y\rangle$ is nonempty. Further, for every $a \in T_{x} \cap\langle Y\rangle$, we have that a belongs to the nucleus of a unique $X$-ovoid $Q$ through $x$.

Proof Since $N<N(n, k)$, and since every 3-dimensional subspace of $\mathscr{P}$ gives rise to a local Veronesean subcap which spans a space of dimension $N(3, k)$ (by Lemma 4.8), there exists a minimal number $j, 3<j \leq n$, such that some $j$-dimensional subspace $U \subseteq X$ of $\mathscr{P}$ gives rise to a local Veronesean subcap which spans a subspace $\langle U\rangle$ of dimension strictly less than $N(j, k)$ (and so every $(j-1)$-dimensional subspace of $\mathscr{P}$ gives rise to a local Veronesean subcap which spans a subspace of dimension exactly $N(j-1, k)$ ).

Let $U_{1}$ be any $(j-1)$-dimensional subspace in $\mathscr{P}$ of $U$; then by the choice of $j$ we have $\operatorname{dim}\left\langle U_{1}\right\rangle=N(j-1, k)$. Let $x \in U \backslash U_{1}$. Since $\operatorname{dim}\langle U\rangle<N(j, k)$ and $\operatorname{dim} T_{x}(U)=k j$, a dimension argument implies that we can find a point $a \in T_{x}(U) \cap\left\langle U_{1}\right\rangle$. Choose a hyperplane $Y$ of $\mathscr{P}$ 
arbitrarily but such that $x \notin Y \supseteq U_{1}$. Since $T_{x}(U) \subseteq T_{x}(X)$ and $\left\langle U_{1}\right\rangle \subseteq\langle Y\rangle$, we have $a \in T_{x}(X) \cap$ $\langle Y\rangle$.

Since $a \in T_{x}$, Lemma 4.2 implies that the line $\langle a, x\rangle$ is contained in a unique $\xi \in \Xi$, and it is tangent to the $X$-ovoid $Q=X \cap \xi$. Set $y=Q \cap Y$. Since $Y$ is full, the subspace $\langle Y\rangle$ does not contain further points of $Q$ (only $y$ ), hence the line $\langle a, y\rangle$ is also tangent to $Q$. This implies that $a$ belongs to the nucleus of $Q$.

Let $N_{y}(Y)$ be the union of the nuclei of the ovoids $X(\xi), \xi \in \Xi$, with $y \in X(\xi) \subseteq Y$, and where $Y$ is a hyperplane of $\mathscr{P}$. Now, we may assume by induction on $n$ that $Y$, endowed with all elliptic spaces $\xi \in \Xi$ such that $X(\xi) \subseteq Y$, is projectively equivalent to a legal projection of the standard local Veronesean cap $\mathscr{V}_{n-1}(\mathbb{K}, \mathbb{A})$.

Lemma 4.14 The set $N_{y}(Y)$ as defined above is a subspace of dimension $k n-k-1$.

Proof The set $N_{y}(Y)$ is contained in $T_{y}(Y)$, which has dimension $k n-k$ by Lemma 4.2. Hence $T_{y}(Y)$ is an isomorphic projection of the corresponding tangent space of the standard local Veronesean cap.

Now, on the one hand, again by Lemma 4.2, every line $L$ in $T_{y}(Y)$ through $y$ is tangent to some $X$-ovoid $Y \cap \zeta$, with $\zeta \in \Xi$ and $\zeta \cap X \subseteq Y$. Hence $L$ contains a unique point of $N_{y}(Y)$. On the other hand, by Corollary 5.6, $N_{y}(Y)$ is contained in the projection of the nucleus subspace of the standard local Veronesean cap, which is a subspace not containing $y$ and hence intersecting $T_{y}(Y)$ in a proper subspace $P \not \supset y$. But $P$ contains a point of every line through $y$, hence it is a hyperplane and coincides with $N_{y}(Y)$.

Let $Q, x, y, Y$ and $a$ be as in Lemma 4.13. We claim that $a \notin N_{y}(Y)$. Indeed, otherwise $a \in\left\langle Q^{\prime}\right\rangle$, with $Q^{\prime}$ an $X$-ovoid contained in $Y$. Since also $a \in\langle Q\rangle$, Axiom (V2) leads to $a \in X$, contradicting the assumption that all hyperplanes of $\mathscr{V}$, and hence also $Y$, are full.

We now show that there is a natural bijection between the points of $\left\langle N_{y}(Y), a\right\rangle \backslash N_{y}(Y)$ and the $X$-ovoids not contained in $Y$ but containing $y$. We need a slightly more general result, however.

Lemma 4.15 Let $U$ be a subspace of $\mathscr{P}$. Let $y \in U$ and $x \notin U, x \in X$. Set $Q=X([x, y])$. Let a be any point of $T_{x}(Q) \cap T_{y}(Q)$. Let $U^{\prime}$ be the subspace of $\mathscr{P}$ generated by $U$ and $x$. Then every point of $\left\langle a, N_{y}(U)\right\rangle \backslash N_{y}(U)$ belongs to the nucleus of some $X$-ovoid $Q^{\prime} \subseteq U^{\prime}$, with $y \in Q^{\prime}$ and $Q^{\prime}$ not contained in $U$. Conversely, the nucleus of every $X$-ovoid $Q^{\prime} \subseteq U^{\prime}$, with $y \in Q^{\prime}$ and $Q^{\prime}$ not contained in $U$, contains a unique point of $\left\langle a, N_{y}(U)\right\rangle \backslash N_{y}(U)$.

Proof Let $a^{\prime}$ be a point of $\left\langle a, N_{y}(U)\right\rangle \backslash N_{y}(U)$ and we may assume $a \neq a^{\prime}$. The line $\left\langle a, a^{\prime}\right\rangle$ intersects $N_{y}(U)$ in a point $b$, which belongs to the nucleus space of a unique $X$-ovoid in $U$, say $b \in \zeta \in \Xi$, with $X(\zeta) \subseteq U$. Let $\xi=[x, y]$ correspond to the line $L$ of $\mathscr{P}$, and let $\zeta$ correspond to the line $M$ of $\mathscr{P}$. The lines $L$ and $M$ define a unique plane $\pi$ of $\mathscr{P}$, which induces on $X$ a global Veronesean cap. Both $a$ and $b$ belong to the nucleus space of that cap, and to the tangent space at $y$. Hence also $a^{\prime}$ belongs to both spaces. So there is some $X$-ovoid $Q^{\prime} \subseteq \pi$, with $y \in Q^{\prime}$ and with $a^{\prime} \in\left\langle Q^{\prime}\right\rangle$. Then $Q^{\prime} \subseteq U^{\prime}$, but $Q^{\prime}$ does not belong to $U$.

Conversely, let $Q^{\prime} \subseteq U^{\prime}$ be an $X$-ovoid such that $U \cap Q^{\prime}=\{y\}$. We may assume $Q^{\prime} \neq Q$. The plane $\pi$ of $\mathscr{P}$ containing the $X$-ovoids $Q$ and $Q^{\prime}$ has a line $M$ contained in $U$; suppose $\zeta \in \Xi$ 
corresponds to $M$. The nucleus subspace $B$ of the $X$-ovoid $X(\zeta)$ is contained in $N_{y}(U)$. The tangent space $T_{y}(\pi)$ in $y$ of the global Veronesean cap defined by $\pi$ has dimension $2 k$ and intersects the nucleus subspace $\mathrm{N}(\pi)$ of $\pi$ in a subspace $N_{y}(\pi)$ of dimension $2 k-1$. If $A, A^{\prime}$ are the nucleus subspaces of $X(\xi)$ and $X\left(\xi^{\prime}\right)$, respectively, then the $(k-1)$-spaces $B, A, A^{\prime}$ are all contained in $N_{y}(\pi)$. Hence, by a dimension argument, the subspace $\langle B, a\rangle$ intersects $A^{\prime}$ in at least a point $a^{\prime}$, but not in a line as that line would intersect $B$ and lead to the contradiction that $A^{\prime} \cap B$ would be nonempty. Hence $a^{\prime}$ is the unique point we want.

Remark 4.16 We use the notation of the previous lemma. The points of $\left\langle N_{y}(U), a\right\rangle \backslash N_{y}(U)$ together with the $k$-spaces of $\left\langle N_{y}(U), a\right\rangle$ not contained in $N_{y}(U)$ but intersecting $N_{y}(U)$ in the nucleus subspace of some $X$-ovoid in $U^{\prime}$, is an André/Bruck-Bose representation $[1,3]$ of the (point-line part of the) affine space whose points are the lines of $\mathscr{P}$ through $y$ contained in $U^{\prime}$ but not in $U$ and whose lines are the planes of $\mathscr{P}$ through $y$ contained in $U^{\prime}$ but not in $U$. This is easily seen using the previous lemma.

Standing notation. From now on we use the notation $Y, Q, x, y$ and $a$ as in the statement and the proof of Lemma 4.13 for the rest of this section.

In order to start the lifting procedure, we only have to find an appropriate Hermitian pencil $\mathscr{U}$ of hyperplanes of $\mathscr{P}$ which generates $\mathbb{P}(V)$. We accomplish this in the following lemma.

Lemma 4.17 With the above notation, let $Z \subseteq Y$ correspond to an $(n-2)$-dimensional space of $\mathscr{P}$, with $y \notin Z$. Let $H$ be any hyperplane of $\langle Q\rangle=[x, y]$ containing $y$ and not containing $a$. Then $\operatorname{dim}\langle Q \cap H\rangle=k$ and $\mathscr{U}=\mathscr{U}(Z, Q \cap H)$ spans $\mathbb{P}(V)$.

Proof Note that, since $H$ does not contain $a$, it is not a tangent hyperplane of $Q$. Hence $\langle Q \cap H\rangle$ is a hyperplane of $\langle Q\rangle$, so that $\mathscr{U}$ is well-defined as a Hrmitian pencil of yperplanes.

Note also that $\langle\mathscr{U}\rangle$ contains $a$, as $a \in\langle Y\rangle$ and $Y \subseteq \mathscr{U}$. Hence, as $[x, y]=\langle H, a\rangle$, we deduce $Q \subseteq\langle\mathscr{U}\rangle$. Now the rest of the proof is completely similar to the fourth paragraph of the proof of Lemma 4.11.

We are now ready to prove the final step.

Lemma 4.18 Let $(X, \Xi)$ be a local Veronesean cap in $\mathbb{P}(V)$ with index $n$ and subdimension $k$. If $Y \subseteq X$ is a full hyperplane of $\mathscr{P}$, and if $\langle Y\rangle$ contains some point a which belongs to the nucleus subspace of an $X$-ovoid $X \cap \xi$, with $\xi \in \Xi$ and $Y \cap \xi=\{y\}$, then $X$ is the image under a central projection $\rho$ of a local Veronesean cap $X^{\prime}$ (with corresponding projective geometry $\left.\mathscr{P}^{\prime}\right)$ spanning some $(N+1)$-dimensional projective space.

Proof We embed $\mathbb{P}(V)$ as a hyperplane in a projective space $\mathbb{P}\left(V^{\prime}\right)$ of dimension $N+1$, and we choose a point $c$ in $\mathbb{P}\left(V^{\prime}\right)$ not in $\mathbb{P}(V)$. On the line $\langle c, a\rangle$ we choose a point which we denote by $a^{*}$, and we choose it such that $a \neq a^{*} \neq c$. Set $N_{y}=N_{y}(Y)$. It follows that the space $\left\langle N_{y}, a\right\rangle$ is the bijective projection from $c$ onto $\mathbb{P}(V)$ of the space $\left\langle N_{y}, a^{*}\right\rangle$. For any point $e \in\left\langle N_{y}, a\right\rangle \backslash N_{y}$, we denote by $e^{*}$ the unique point of $\langle e, c\rangle \cap\left\langle N_{y}, a^{*}\right\rangle$. This way, we see that $a$ and $e$ play the same role. 
Recall that $a$ belongs to the nucleus subspace of the $X$-ovoid $Q=X([x, y])$. Let $H$ be a hyperplane of $[x, y]$ containing $y$ and not containing $a$, as in the statement of the lemma.

We pick an $(n-2)$-space $Z$ of $\mathscr{P}$ contained in $Y$, but not containing $y$. We define for each point $u \in \mathscr{U}=\mathscr{U}(Z, H \cap Q)$ the point $u^{\theta}$ as $u$ itself. Now let $w \in X \backslash \mathscr{U}$; in particular $w \notin Y$. By Lemma 4.15 (putting $U=Y$ ), there is a unique point $e$ in $T_{w} \cap\left\langle N_{y}, a\right\rangle$. Denote $Q_{e}=X([w, y])$. We claim that $\left\langle Q_{e} \cap \mathscr{U}\right\rangle$ does not contain $e$. Indeed, since $Q$ and $Q_{e}$ intersect in $y$, we can include them in a unique (common) global Veronesean cap, say with point set $\pi$. Let $Q_{Y}$ be the $X$-ovoid corresponding to the line $\pi \cap Y$ of $\mathscr{P}$. Then $\pi \cap \mathscr{U}$ is a Hermitian pencil of lines and generates a hyperplane of $\pi$ not containing $a$, but containing the nucleus subspace $N^{\prime}$ of $Q_{Y}$. If $\langle\pi \cap \mathscr{U}\rangle$ would contain $e$, then also $\left\langle N^{\prime}, e\right\rangle \ni a$, a contradiction. Hence $e$ lies outside $\left\langle Q_{e} \cap \mathscr{U}\right\rangle$ and the claim is proved.

Hence $Q_{e} \cap \mathscr{U}$ defines a hyperplane section $H_{e} \cap Q_{e}$, with $H_{e}$ a $k$-space of $[w, y]$ not containing $e$. Then the spaces $[w, y]=\left\langle H_{e}, e\right\rangle$ and $\left\langle H_{e}, e^{*}\right\rangle$ are a central projection of each other from $c$, and hence the $X$-ovoid $Q_{e}$ is the central projection of an ovoid $Q_{e}^{*} \subseteq\left\langle H_{e}, e^{*}\right\rangle$. Hence, for each point $p \in Q_{e}$, there is a unique point $p^{\theta} \in Q_{e}^{*}$ with $p^{\theta} \in\langle c, p\rangle$, and $p^{\theta}=p$ if and only if $p \in \mathscr{U}$. Now put $X^{\theta}=\left\{z^{\theta}: z \in X\right\}$.

Let $S \subseteq X$ correspond to the point set of a 3 -space of $\mathscr{P}$ such that $S \cap Z$ is a line of $\mathscr{P}$ and $y \in S$. Then $\mathscr{U} \cap S$ is a Hermitian pencil of planes in $S$. Lemma 4.8 and the definition of Hermitian pencil of hyperplanes assert that $\mathscr{U} \cap S$ spans a hyperplane of $\langle S\rangle$. Set $\pi=S \cap Y$. Let $N_{y}(\pi)$ be the union of all nucleus subspaces of ovoids through $y$ in $\pi$ (then by Lemma 4.14, $N_{y}(\pi)$ is a subspace of dimension $2 k-1$ ). Let $O$ be some $X$-ovoid in $S$ not entirely contained in $\pi$ and with $y \in O$. Then, by Lemma 4.15 applied for $U=Y$, there is a point $e$ in the nucleus of $O$ which is contained in $\left\langle N_{y}, a\right\rangle \backslash N_{y}$. Then, again by Lemma 4.15 now applied to $U=\pi$ and $U^{\prime}=S$, there is a natural bijective correspondence between the points of $\left\langle N_{y}(\pi), e\right\rangle \backslash N_{y}(\pi)$ and the ovoids through $y$ entirely contained in $S$ but not in $Y$. By construction, $S^{\theta}:=\left\{s^{\theta}: s \in S\right\}$ is contained in $\left\langle\mathscr{U} \cap S, e^{*}\right\rangle$. The latter does not contain $c$ as it would otherwise also contain $\left\langle N_{y}(\pi), e\right\rangle$, and hence $\langle S\rangle$, implying it would have dimension $N(3, k)+1$, a contradiction. Hence $S^{\theta}$ is the bijective projection of $S$ from $c$. This implies that $S^{\theta}$, endowed with all projections of ovoids, is a local Veronesean cap of index 3 isomorphic to $\mathscr{V}_{3}(\mathbb{K}, \mathbb{A})$.

Since every line $L$ of $\mathscr{P}$, not contained in $Y$, can be put into a 3 -space of $\mathscr{P}$ containing also $y$, and intersecting $Z$ in a line, we see that, $L^{\theta}:=\left\{u^{\theta}: u \in L\right\}$ is an ovoid. Putting $\Xi^{\theta}=\left\{\left\langle L^{\theta}\right\rangle\right.$ : $L \in \mathscr{L}\}$, the pair $\left(X^{\theta}, \Xi^{\theta}\right)$ already satisfies (V1).

Now we consider and fix any point $x \in X \backslash \mathscr{U}$. Let $u$ be any other point. We can put $x, u$ and $y$ in a 3-space $S$ of $\mathscr{P}$ intersecting $Z$ in a line. The above argument implies that $S^{\theta}$ is contained in $\left\langle S \cap \mathscr{U}, x^{\theta}\right\rangle$, and so $u^{\theta}$ is the unique point in $\langle c, u\rangle \cap\left\langle S \cap \mathscr{U}, x^{\theta}\right\rangle$. Consequently, we can define $\theta$ starting from $x^{\theta}$. Notice this is independent of $x$ ! Hence, we can now repeat the arguments in the proof of Lemma 4.11 and conclude that $\left(X^{\theta}, \Xi^{\theta}\right)$ is a local Veronesean cap in $\mathbb{P}\left(V^{\prime}\right)$. Consequently, $(X, \Xi)$ is the image of $\left(X^{\theta}, \Xi^{\theta}\right)$ under the central projection $\rho$ with center $c$, where $\left(x^{\theta}\right)^{\rho}=x$, for all $x \in X$. Hence, putting $X^{\theta}=X^{\prime}$, the lemma is proved.

End of the proof of the Main Result 1. The previous lemma implies that, if every hyperplane of $\mathscr{P}$ is full for $(X, \Xi)$, and if $N<N(n, k)$, then $(X, \Xi)$ is the projection of another local Veronesean cap of index $n$ and subdimension $k$ for which the associated projective space is isomorphic to $\mathscr{P}$. Applying this again when $N+1<N(n, k)$, and then again until we reach the dimension 
$N(n, k)$, proves the Main Result for the inseparable case.

\section{Induced projective representations}

We now proceed with proving Main Result 2.

Let $\mathscr{V}=\mathscr{V}_{n}(\mathbb{K}, \mathbb{A})$ be the standard local Veronesean cap of index $n \geq 3$ (for $n=2$, see [9], Section 7) associated to the associative quadratic division algebra $\mathbb{A}$ over the field $\mathbb{K}$, in the finite-dimensional projective space $\mathbb{P}(V)$, with $V$ as in Section 2. A collineation of a projective space is a permutation of its point set inducing a permutation of its line set. An elation of a projective space is a collineation $\varphi(p, H)$ that fixes a hyperplane $H$ pointwise (and $H$ is called the axis), and also fixes all hyperplanes (globally) through a certain point $p \in H$ (and $p$ is called the center). Let $G$ be the so-called little projective group of $\mathscr{P}=\mathscr{P}^{n}(\mathbb{A})$, i.e., $G$ is the collineation group generated by all elations of $\mathscr{P}$. We can identify the point set of $\mathscr{P}$ with the point set $X$ of $\mathscr{V}$. Hence we can view $G$ as a set of permutations of $\mathscr{V}$ preserving the family of $X$-ovoids.

In this section (see Subsection 5.1) we show that $G$ is induced by a collineation group $G^{\dagger}$ of $\mathbb{P}(V)$ and that the $G$-action on $\mathscr{V}$ is rigid in the sense that each element of $G$ unique extends to a collineation of $\mathbb{P}(V)$. Hence we obtain a projective representation of $G$ in $\mathbb{P}(V)$. We determine all invariant subspaces of that projective representation, and we identify the projective representations induced in the invariant subspaces and the corresponding quotients (see the end of Subsection 5.2). Our results show that $G$ acts irreducibly on $\mathbb{P}(V)$, except in the purely inseparable case and the case $\mathbb{K}=\mathbb{A}$ with char $\mathbb{K}=2$, where the action is nevertheless indecomposable.

The latter case gives rise to some special geometric features. In this case, we not only have the standard local Veronesean cap $\mathscr{V}$, but there is a nucleus subspace in which we can see a representation of the line Grassmann space corresponding to $\mathscr{P}$. The projection of the standard local Veronesean cap from the nucleus space yields the embedding of $\mathscr{P}$ into an $n$-dimensional subspace of $\mathbb{P}(V)$ corresponding to the inclusion $\mathbb{A}^{2} \subseteq \mathbb{K}$ of coordinatizing fields.

In the first part of Subsection 5.2, we show that $G$ is never induced in any proper legal projection of $\mathscr{V}$ by the collineation group of the ambient projective space. In fact, we show a slightly stronger result except if $\mathbb{K}=\mathbb{A}=\mathbb{F}_{2}$, see Theorem 5.11. Here, we also include the case $n=2$ in the stronger result.

\section{1 (Ir)reducibility and indecomposability}

From now on, we are given the standard local Veronesean cap $\mathscr{V}=\mathscr{V}_{n}(\mathbb{K}, \mathbb{A})$ in $\mathbb{P}(V)$, with little projective group $G$. For the time being, we assume $n \geq 3$.

Proposition 5.1 With the above notation, $G$ is induced by a collineation group $G^{\dagger}$ of $\mathbb{P}(V)$. More precisely, the action of $G$ on $\mathscr{V}$ is rigid yielding a collineation group $G^{\dagger} \cong G$ of $\mathbb{P}(V)$.

Proof Since $G$ is generated by all elations $\varphi(p, H)$, with $p$ ranging over the points and $H$ ranging over the hyperplanes through $p$, it suffices to consider, with respect to an arbitrary 
coordinatization and for all $i, j \in\{0,1, \ldots, n\}$, the central collineations $\varphi\left(p_{i}, H_{j}\right), i \neq j$, where $p_{i}$ is the point with only nonzero coordinate at position $i$, and $H_{j}$ consist of all points whose coordinate at position $j$ is zero (indeed, one checks that the group generated by these elations acts transitively on the set of all pairs $(p, H)$, where $p$ is a point and $H$ a hyperplane with $p \in H)$. Without loss of generality we may take $j=0$. For $i$ we could take, also without loss, any of $1,2, \ldots, n$, but we will later need the general form of any elation with axis $H_{0}$ and center an arbitrary point $c$ of $H_{0}$.

Let $c$ be given by $\left(0, \gamma_{1}, \ldots, \gamma_{n}\right)$, with $\gamma_{i} \in \mathbb{A}, i=1,2, \ldots, n$. Let $\theta_{0, c}$ be the collineation of $\mathbb{P}(V)$ induced by the following $\mathbb{K}$-linear map of $V$ (which we also denote by $\theta_{0, c}$ ), with obvious notation $(i, j, k \in\{1,2, \ldots, n\}, j<k)$.

$$
\begin{aligned}
\theta_{0, c}: V \rightarrow V: \quad & \left(y_{0}, y_{i} ; y_{0, i}, y_{j, k}\right) \mapsto \\
& \left(y_{0}, y_{i}+\gamma_{i} \gamma_{i}^{\sigma} y_{0}+\gamma_{i} y_{0, i}+y_{0, i}^{\sigma} \gamma_{i}^{\sigma} ; y_{0, i}+\gamma_{i}^{\sigma} y_{0}, y_{j, k}+\gamma_{j} y_{0, k}+y_{0, j}^{\sigma} \gamma_{k}^{\sigma}+\gamma_{j} \gamma_{k}^{\sigma} y_{0}\right) .
\end{aligned}
$$

One easily checks that $\theta_{0, c}$ preserves $\mathscr{V}$ and acts on $\mathscr{P}$ as

$$
\left(x_{0}, \cdots, x_{n}\right) \mapsto\left(x_{0}, x_{1}+\gamma_{1} x_{0}, \cdots, x_{n}+\gamma_{n} x_{0}\right),
$$

which is a generic elation with axis $H_{0}$ and center $c$ (to obtain all elations with given center $c$ one has to consider all coordinate tuples representing $c$, i.e., consider all nonzero scalar $\mathbb{A}$-multiples of $\left.\left(0, \gamma_{1}, \ldots, \gamma_{n}\right)\right)$. Note that $\theta_{0, c}$ heavily depends on the given coordinates of $c$; hence the $c$ in the index is to be read as the corresponding $(n+1)$-tuple rather than as the point $c$.

If we denote for a given elation $\eta$ of $\mathscr{P}$ the corresponding collineation of $\mathbb{P}(V)$ preserving $\mathscr{V}$ as found above by $\bar{\eta}$, then it remains to show that the $\bar{\eta}$ really generate a group isomorphic to $G$. To that aim it suffices to prove that every collineation $\varphi$ of $\mathbb{P}(V)$ fixing $\mathscr{V}$ pointwise is the identity.

To that aim, consider the three hyperplanes $H_{0}, H_{1}, H_{2}$ of $\mathscr{P}$. Then, since $\left\langle H_{i}\right\rangle \cap\left\langle H_{j}\right\rangle \neq \emptyset$, for $i, j \in\{0,1,2\}$, the collineation $\varphi$ fixes $\left\langle H_{0}, H_{1}, H_{2}\right\rangle$ pointwise. Now, $T_{x} \subseteq\left\langle H_{1}, H_{2}\right\rangle$, for every $x \in H_{1} \cap H_{2}$, and so Lemma 4.4 applied to $p_{0}$ and $H_{0}$ implies that $\left\langle H_{0}, H_{1}, H_{2}\right\rangle=\mathbb{P}(V)$ and we are done.

Lemma 5.2 Let $Y$ be the subset of $\mathscr{V}$ corresponding to a hyperplane of $\mathscr{P}$. Then there is a unique hyperplane $\mathscr{H}_{0}$ of $\mathbb{P}(V)$ intersecting $\mathscr{V}$ in $Y$, i.e., $\mathscr{H}_{0} \cap \mathscr{V}=Y$.

Proof This lemma can be shown in a completely geometric way using our axioms, but since below we need an algebraic expression anyway, we establish this in an algebraic way. Without loss of generality, we may assume that $Y$ corresponds to $H_{0}$ (with above notation). The subspace $\langle Y\rangle$ of $\mathbb{P}(V)$ generated by $Y$ is given by the equations $y_{0}=y_{0, i}=0, i \in\{1,2, \ldots, n\}$. Let $\mathscr{H}_{0}$ be the hyperplane of $\mathbb{P}(V)$ with equation $y_{0}=0$. Then $\mathscr{H}_{0} \cap \mathscr{V}=Y$. Now let $\mathscr{H}^{*}$ be a hyperplane of $\mathbb{P}(V)$ containing $Y$, but distinct from $\mathscr{H}_{0}$. Then $\mathscr{H}^{*}$ contains a point $p$ with coordinates $\left(1, y_{i} ; y_{j, k}\right)_{1 \leq i \leq n ; 0 \leq j<k \leq n}$. Define the point $\left(1, y_{0, k}^{\sigma}\right)_{1 \leq k \leq n}$ of $\mathscr{P}$. The corresponding point $p^{*}$ of $\mathscr{V}$ has coordinates

$$
\left(1, y_{0, i} y_{0, i}^{\sigma} ; y_{0, j}, y_{0, k}^{\sigma} y_{0, \ell}\right)_{1 \leq i \leq n ; 1 \leq j \leq n ; 1 \leq l<\ell \leq n},
$$

and hence we see that the line $\left\langle p, p^{*}\right\rangle$ intersects $\langle Y\rangle$ nontrivially. Hence $\mathscr{H}^{*} \supseteq\langle Y, p\rangle$ contains $p^{*} \in \mathscr{V}$. It follows that $\mathscr{H}_{0}$ is the unique hyperplane intersecting $\mathscr{V}$ precisely in $Y$. 
With the notation of the previous lemma we call $\mathscr{H}_{0}$ a tangent hyperplane (tangent at $Y$ ). We have the following result.

Lemma 5.3 With the above notation, the (common) intersection of all tangent hyperplanes is empty if and only if $\mathbb{A}$ is not a purely inseparable field extension of $\mathbb{K}$ and $\mathbb{K} \neq \mathbb{A}$ if char $\mathbb{K}=2$. If it is not empty, it is a subspace of dimension $k \frac{n(n+1)}{2}-1$.

Proof Suppose first that $\mathbb{A}^{2} \subseteq \mathbb{K}$ and char $\mathbb{K}=2$. Let $H$ be a tangent hyperplane and let the equation of the corresponding hyperplane of $\mathscr{P}$ be $\sum_{i=1}^{n} a_{i} x_{i}=0$, where $a_{i} \in \mathbb{K}, i=0,1, \ldots, n$. Then one can verify that $H$ has the equation $\sum_{i=1}^{n} a_{i}^{2} y_{i}=0$. Consequently $H$ contains the subspace $N$ with equations $y_{0}=y_{1}=\cdots=y_{n}=0$. Hence $N$ is the common intersection of all tangent hyperplanes and has dimension $k \frac{n(n+1)}{2}-1$.

Now suppose $\mathbb{A}^{2}$ is not contained in $\mathbb{K}$ or char $\mathbb{K} \neq 2$. Let $p=\left(y_{i} ; y_{j, k}\right)_{0 \leq i \leq n ; 0 \leq j<k \leq n}$ be contained in every tangent hyperplane. Since the tangent hyperplane $\mathscr{H}_{i}$ corresponding to the hyperplane of $\mathscr{P}$ with equation $x_{i}=0, i \in\{0,1, \ldots, n\}$, has equation $y_{i}=0$ in $\mathbb{P}(V)$, we see that $y_{0}=y_{1}=\cdots=y_{n}=0$. Without loss of generality we may assume that $y_{0,1} \neq 0$. If char $\mathbb{K} \neq 2$, then we define $\gamma_{1}=y_{0,1}^{\sigma}$; if char $\mathbb{K}=2$, then $\sigma$ is not the identity, we select $a \in \mathbb{A}$ with $a \neq a^{\sigma}$ and we define $\gamma_{1}=a y_{0,1}^{-1}$. Putting $c=\left(0, \gamma_{1}, 0, \ldots, 0\right)$, we easily see that $\theta_{0, c}$ maps $p$ to a point with nonzero second coordinate, hence not belonging to the tangent hyperplane $\mathscr{H}_{1}$. Hence $p$ does not belong to the tangent hyperplane $\theta_{0, c}^{-1}\left(\mathscr{H}_{1}\right)$.

The common intersection of all tangent hyperplanes, if nonempty, will be called the nucleus of $\mathscr{V}$ and denoted by $\mathrm{N}(\mathscr{V})$.

Proposition 5.4 Suppose that $\mathbb{A}$ is not a purely inseparable field extension of $\mathbb{K}$ and $\mathbb{K} \neq \mathbb{A}$ if char $\mathbb{K}=2$. Then $G^{\dagger}$ acts irreducibly on $\mathbb{P}(V)$.

Proof We have to show that the orbit $p^{G^{\dagger}}$ generates $\mathbb{P}(V)$, for every point $p$ of $\mathbb{P}(V)$. If $p \in \mathscr{V}$, then this follows from the fact that $G^{\dagger}$ acts transitively on $\mathscr{V}$. Hence we may suppose that $p$ does not belong to $\mathscr{V}$. By our assumptions, $p$ is not contained in every tangent hyperplane. Without loss of generality, this means that we may assume that the first coordinate of $p$ in $\mathbb{P}(V)$ is equal to 1 . Let $p$ be represented by $\left(1, y_{i} ; y_{j, k}\right)_{1 \leq i \leq n ; 0 \leq j<k \leq n}$.

Suppose first that $|\mathbb{K}|>2$. Let $t \in \mathbb{K} \backslash\{0,1\}$ be arbitrary and let $c$ be the point of $\mathscr{P}$ having coordinates $(0, t, 0, \ldots, 0)$. We compute the coordinates of the point $q$ on the line $\left\langle p, \theta_{0, c}(p)\right\rangle$ obtained by adding $t-1$ times the original coordinate tuple of $p$ to the coordinate type of $\theta_{0, c}(p)$ obtained above. We obtain for $q$ the coordinates

$$
\left(t, t y_{1}+t^{2}+t y_{0,1}+t y_{0,1}^{\sigma}, t y_{i} ; t y_{0,1}+t, t y_{0, i}, t y_{1, j}+t y_{0, j}, t y_{k, \ell}\right)_{2 \leq i \leq n ; 2 \leq j \leq n ; 2 \leq k<\ell \leq n} .
$$

Now we put $c^{\prime}=(0,-1,0, \ldots, 0)$ and we compute $\theta_{0, c^{\prime}}(q)$. We obtain the following coordinate tuple:

$$
\left(t, t y_{1}+t^{2}-t, t y_{i} ; t y_{0,1}, t y_{0, i}, t y_{1, j}, t y_{k, \ell}\right)_{2 \leq i \leq n ; 2 \leq j \leq n ; 2 \leq k<\ell \leq n} .
$$

If we connect this point with $p$ and intersect the obtained line with the hyperplane with equation $Y_{0}=0$, then we see that the point $r=\left(0, t^{2}-t, 0, \ldots, 0 ; 0, \ldots, 0\right)$ belongs to the span of $p^{G^{\dagger}}$. 
Since $r$ also belongs to $\mathscr{V}$, and the orbit of $r$ generates $\mathbb{P}(V)$ by the first lines of the proof, we conclude $\mathbb{P}(V)=\langle\mathscr{V}\rangle \subseteq\left\langle p^{G^{\dagger}}\right\rangle$.

Now suppose $|\mathbb{K}|=2$. Our assumptions imply that $\mathbb{A}$ is the field of order 4 ; set $\mathbb{A}=\{0,1, \varepsilon, \varepsilon+$ $1\}$. We choose $t \in \mathbb{A} \backslash\{0\}$ arbitrary. Define $c=(0, t, 0, \ldots, 0)$ again. Then the "third point" $q_{t}$ on the line joining $p$ with $\theta_{0, c}(p)$ has coordinates

$$
\left(0,1+t y_{0,1}+t^{\sigma} y_{0,1}^{\sigma}, 0, \ldots, 0 ; t^{\sigma}, 0, \ldots, 0, t y_{0, i}, 0, \ldots, 0\right)_{2 \leq i \leq n},
$$

where $t y_{0, i}$ is positioned in the entry $(1, i)$, for all $i \in\{2,3 \ldots, n\}$. But now we see that $q_{1}+q_{\varepsilon}+$ $q_{\varepsilon+1}$, which belongs to $\left\langle p^{G^{\dagger}}\right\rangle$, is exactly the point $p_{1}=(0,1,0, \ldots, 0)$ and hence belongs to $\mathscr{V}$. As in the first part of the proof, we conclude $\mathbb{P}(V)=\langle\mathscr{V}\rangle \subseteq\left\langle p^{G^{\dagger}}\right\rangle$.

Proposition 5.5 Suppose that $\mathbb{A}$ is a purely inseparable field extension of $\mathbb{K}$ or $\mathbb{K}=\mathbb{A}$ and char $\mathbb{K}=2$. Then $\mathrm{N}(\mathscr{V})$ is the only proper subspace of $\mathbb{P}(V)$ that is invariant under $G^{\dagger}$. Hence the action of $G^{\dagger}$ on $\mathbb{P}(V)$ is indecomposable.

Proof We first show that the orbit $p^{G^{\dagger}}$ generates $\mathbb{P}(V)$, for every point $p$ of $\mathbb{P}(V) \backslash N(\mathscr{V})$. Since $p \notin N(\mathscr{V})$, we may assume by the proof of Lemma 5.3 that, without loss of generality, the first coordinate of $p$ in $\mathbb{P}(V)$ is equal to 1 . A similar calculation as performed in the first part of the proof of Proposition 5.4 shows that, if $|\mathbb{K}|>2$, the point $p_{1}=(0,1,0, \ldots, 0)$ is contained in both $p^{G^{\dagger}}$ and $\mathscr{V}$, implying $\left\langle p^{G^{\dagger}}\right\rangle=\mathbb{P}(V)$.

Now suppose $|\mathbb{K}|=2$. Then $\mathbb{A}=\mathbb{K}$. Let $p$ again have coordinates $\left(1, y_{i} ; y_{j, k}\right)_{1 \leq i \leq n ; 1 \leq j<k \leq n}$. We may suppose $y_{0, i}=0$, for $i \in\{2, \ldots, n\}$, since the mapping $\theta_{0, c}$, with $c=\left(0,0, y_{0,2}, y_{0,3}, \ldots, y_{0, n}\right)$ interchanges 0 and 1 in the position $(0, i)$. Define $c^{\prime}=(0,1,0, \ldots, 0)$. Then the "third point" $q$ on the line joining $p$ with $\theta_{0, c^{\prime}}(p)$ has coordinates

$$
(0,1,0, \ldots, 0 ; 1,0, \ldots, 0)_{2 \leq i \leq n} .
$$

The point $q$ belongs to the subspace generated by the (standard) global Veronesean $\mathscr{V}_{012}$ induced by the plane spanned by $p_{0}, p_{1}$ and $p_{2}$ in $\mathscr{P}$, but not to its nucleus $\mathrm{N}\left(\mathscr{V}_{012}\right)$. The stabiliser of $\mathscr{V}_{012}$ in $G^{\dagger}$ only stabilises $\mathrm{N}\left(\mathscr{V}_{012}\right)$, hence $\left\langle\mathscr{V}_{012}\right\rangle \subseteq\left\langle p^{G^{\dagger}}\right\rangle$, In particular, $\left\langle p^{G^{\dagger}}\right\rangle$ contains elements of $\mathscr{V}$ and hence contains $\mathscr{V}$ and we conclude $\left\langle p^{G^{\dagger}}\right\rangle=\mathbb{P}(V)$.

Now let $p=\left(0, \ldots, 0 ; y_{i, j}\right)_{o \leq i<j \leq n}$ belong to $\mathrm{N}(\mathscr{V})$ and let $T_{0} \leq G^{\dagger}$ be the group with generic element $\theta_{0, c}$, with $c=\left(0, \gamma_{1}, \ldots, \gamma_{n}\right), \gamma_{i} \in \mathbb{A}, i=1,2, \ldots, n$. Then $p$ is a fixed point of $T_{0}$ if and only if $y_{0, j}=0$, for all $j \in\{1,2, \ldots, n\}$. So, if $p$ is fixed under $T_{0}$, then $p$ belongs to the nucleus of the standard local Veronesean cap $\mathscr{V}_{0}$ of index $n-1$ generated by the points $p_{1}, p_{2}, \ldots, p_{n}$. If $p$ is not fixed under the group $T_{0}$, then there is some element $\tau \in T_{0}$ such that $p \neq p^{\tau}$ and there is a unique point $q$ on the line $\left\langle p, p^{\tau}\right\rangle$ with first $2 n+1$ coordinates zero (so besides $Y_{i}=$ 0 we also have $Y_{0, j}=0$, for $\left.j \in\{1,2, \ldots, n\}\right)$. Since $q \in\left\langle p^{G^{\dagger}}\right\rangle$, we conclude that $\left\langle p^{G^{\dagger}}\right\rangle \cap$ $\mathrm{N}\left(\mathscr{V}_{0}\right) \neq \emptyset$. Restricting $G^{\dagger}$ to the stabiliser of $\mathscr{V}_{0}$, we find, in a similar way, a point of $\left\langle p^{G^{\dagger}}\right\rangle \cap$ $\mathrm{N}\left(\mathscr{V}_{1}\right)$, where $\mathscr{V}_{1}$ is the standard local Veronesean cap of index $n-2$ generated by the points $p_{2}, p_{3}, \ldots, p_{n}$. Continuing like that, we eventually obtain a nonempty intersection of $\left\langle p^{G^{\dagger}}\right\rangle$ with the (standard) global Veronesean cap $\mathscr{V}_{n-3}$ generated by $p_{n-2}, p_{n-1}, p_{n}$. By Proposition 7.2 
of [9], we have $N\left(\mathscr{V}_{n-3}\right) \subseteq\left\langle p^{G^{\dagger}}\right\rangle$. In particular the subspace of $\mathbb{P}(V)$ consisting of all points $(0, \ldots, 0 ; 0, \ldots, 0, a)$, with $a \in \mathbb{A} \backslash\{0\}$ is entirely contained in $\left\langle p^{G^{\dagger}}\right\rangle$. Likewise this holds for every subspace of $\mathrm{N}(\mathscr{V})$ "generated by one $\mathbb{A}$-entry" in the coordinate representation. This implies that $\mathrm{N}(\mathscr{V}) \subseteq\left\langle p^{G^{\dagger}}\right\rangle$. Equality follows from the first part of the proof and since $G^{\dagger}$ sends nuclei to nuclei.

Corollary 5.6 The subspace of $\mathbb{P}(V)$ generated by the nuclei of the $X$-ovoids coincides with $\mathrm{N}(\mathscr{V})$.

Proof Let $N$ be the subspace generated by the nuclei of the $X$-ovoids of $\mathscr{V}$. Since $N$ is invariant under $G^{\dagger}$, it suffices to prove that $N$ is contained in $\mathrm{N}(\mathscr{V})$.

Let $Y$ be a hyperplane of $\mathscr{P}$ with corresponding tangent hyperplane $\mathscr{H}$, and let $Q$ be an $X$-ovoid with nucleus subspace $S$. If $Q \subseteq Y$, then $S \subseteq \mathrm{N}(\mathscr{V})$. If $Q \cap Y=\{y\}$, then, by the definition of $\mathscr{H}$, we have $\mathscr{H} \cap Q=\{y\}$, and so $\mathscr{H} \cap\langle Q\rangle$ is a hyperplane of $\langle Q\rangle$ tangent to $Q$ at $y$ and hence contains $S$. Consequently $S \subseteq \mathscr{H}$, implying $N \subseteq \mathrm{N}(\mathscr{V})$.

As an application to the existence and uniqueness of the tangent hyperplanes, we can prove the following proposition.

Proposition 5.7 Let $Y$ be a hyperplane of $\mathscr{P}$ with corresponding tangent hyperplane $\mathscr{H}$ and let $x \in X \backslash Y$. Then the projection $\rho$ from $\langle Y\rangle$ onto $T_{x}$ is a bijection from the affine space $\mathscr{P} \backslash Y$ to the affine space $T_{x} \backslash \mathscr{H}$ mapping parallel lines onto parallel $k$-spaces and hence parallel $i$-spaces to parallel $i k$-spaces, $1 \leq i \leq n-1$, where $k=\operatorname{dim}_{\mathbb{K}} \mathbb{A}$.

Proof First we note that Lemmas 4.2, 4.4 and 4.5 imply that $T_{x}$ and $\langle Y\rangle$ are really complementary subspaces of $\mathbb{P}(V)$. This implies that, if $\xi \in \Xi$, then either $\xi \subseteq\langle Y\rangle$ or $\xi \cap\langle Y\rangle$ is a singleton $y \in Y$. Hence, for two distinct points $x_{1}, x_{2} \in X(\xi) \backslash\{y\}$, the line $\left\langle x_{1}, x_{2}\right\rangle$ never meets $\langle Y\rangle$ as no three points of $X$ are collinear. This shows that $\rho$ is injective on $X \backslash Y$. For every point $z \in X \backslash Y$, the point $z^{\rho}$ does not belong to $\mathscr{H}$. We now claim that every point $u \in T_{x} \backslash \mathscr{H}$ is the image under $\rho$ of a point of $X \backslash Y$. Indeed, the line $\langle x, u\rangle$ is, by Lemma 4.2, tangent to some $X$-ovoid $Q \ni x$, which intersects $Y$ is a unique point $y$. Then $\langle y, u\rangle$ is not contained in $\mathscr{H} \supseteq T_{y}(Q)$, hence $\langle y, u\rangle$ intersects $Q$ in a second point $z \neq y$. We obtain $z^{\rho}=u$. Hence $\rho$ is bijective from $X \backslash Y$ onto $T_{x} \backslash \mathscr{H}$.

Set $H_{x}=T_{x} \cap \mathscr{H}$. It is clear that, for every $X$-ovoid $Q$, with $Q \cap Y=\{y\}$, we have $\left\langle Q^{\rho}\right\rangle \cap H_{x}=$ $T_{y}(Q) \cap T_{x}$, and since $\langle Q\rangle \cap\langle Y\rangle=\{y\}$, the subspace $T_{y}(Q) \cap T_{x}$ is $(k-1)$-dimensional, while $\left\langle Q^{\rho}\right\rangle$ is $k$-dimensional.

Let $Q^{\prime}$ be a second $X$-ovoid containing $y$. Then $T_{y}\left(Q^{\prime}\right) \subseteq\left\langle T_{y}(Q), Y\right\rangle$, as follows straight from (V3). Symmetry implies $\left\langle T_{y}(Q), Y\right\rangle=\left\langle T_{y}\left(Q^{\prime}\right), T\right\rangle$. Hence, since $T_{y}(Q) \cap T_{x}=\left\langle T_{y}(Q), Y\right\rangle \cap T_{x}$ (as $\langle Y\rangle$ and $T_{x}$ are disjoint subspaces), we also have

$$
T_{y}\left(Q^{\prime}\right) \cap T_{x}=\left\langle T_{y}\left(Q^{\prime}\right), Y\right\rangle \cap T_{x}=\left\langle T_{y}(Q), Y\right\rangle \cap T_{x}=T_{y}(Q) \cap T_{x} .
$$

This shows that $\rho$ maps parallel lines of the affine space $\mathscr{P} \backslash Y$ onto parallel $k$-spaces of the affine space $T_{x} \backslash H_{x}$. 
Remark 5.8 With the notation of the previous proposition, let $L_{1}, L_{2}$ be two non-parallel lines of the affine space $\mathscr{P} \backslash Y$. Then the images $L_{1}^{\rho}$ and $L_{2}^{\rho}$ span projective subspaces which have at most one point in common, and this point belongs to $T_{x} \backslash \mathscr{H}$. This is easily seen if $L_{1}$ and $L_{2}$ intersect; if they do not, then we consider a translate of $L_{2}$ that intersects $L_{1}$ to reach our assertion.

\subsection{Homogeneity}

Let $G$ be the little projective group of $\mathscr{P}$. Then an injective projection of $\mathscr{V}_{n}(\mathbb{K}, \mathbb{A})$, where we now allow $n=2$, into a subspace $W$ of $\mathbb{P}(V)$ is called $G$-homogeneous if $G$ is induced by the collineations of $W$ stabilizing the said projection. We now prepare the classification of the $G$-homogeneous local Veronesean caps with the following lemmas. We will eventually prove a slightly stronger result by showing that any $G$-homogeneous injective projection of the standard local Veronesean cap is either the cap itself, or its projection from the entire nucleus space, except if $\mathbb{K}=\mathbb{A}=\mathbb{F}_{2}$. This will follow from the fact that, if we project $\mathscr{V}_{n}(\mathbb{K}, \mathbb{A})$ injectively from a subspace $U$ of $\mathbb{P}(V)$ onto a complementary subspace $W$, then a member $\varphi$ of the little projective group of $\mathscr{P}$ acting on the said projection is induced by a collineation of $W$ if and only if the collineation $\varphi^{\prime}$ of $\mathbb{P}(V)$ inducing $\varphi$ on $\mathscr{V}_{n}(\mathbb{K}, \mathbb{A})$ stabilizes $U$. This is our first aim to prove (and will be accomplished in Theorem 5.11).

There is a general result along the same lines in the literature about automorphisms of projections of embedded geometries, see Theorem 13 of [10], but this requires the projected embedded geometry to have enough lines, whereas we do not have any line. The naive idea of adding lines, such as all secants, perhaps works in the case where the nucleus space is trivial, but certainly fails otherwise. Nevertheless, our approach uses the idea of working with the secant lines, but without actually adding them to the geometry.

Recall that two subspaces $A$ and $B$ of another subspace $D$ of a projective space $\mathbb{P}(V)$ are called complementary if $A \cap B=\emptyset$ and $\langle A, B\rangle=D$. A Segre variety $\mathscr{S}$ of type $(1, d)$ is the set of points of a projective space spanning some subspace $D$ of dimension $2 d+1$ which are on a line (such line is called a generator of $\mathscr{S}$ ) that intersects three given mutually complementary subspaces of $D$ (necessarily of dimension $d$ ).

Lemma 5.9 Let $S, S^{\prime}, T^{\prime}$ be three subspaces of a finite-dimensional projective space $U$. Suppose $\operatorname{dim} T^{\prime}=d \geq 0$, both $S$ and $S^{\prime}$ are complementary to $T^{\prime}$ and $U=\left\langle S, S^{\prime}\right\rangle$. Let $S^{\prime \prime}$ be an arbitrary subspace of $S$ complementary to $S \cap S^{\prime}$ in $S$ (then $\left.\operatorname{dim} S^{\prime \prime}=\operatorname{dim} T^{\prime}\right)$. Then each line intersecting all three subspaces $S, S^{\prime}, T^{\prime}$ is contained in a cone $C$ with vertex $S \cap S^{\prime}$ and base a Segre variety $\mathscr{S}$ of type $(1, d)$, containing the subspaces $T^{\prime}, S^{\prime \prime}$ and a subspace $S^{\prime \prime \prime}$ of $S^{\prime}$ complementary to $S \cap S^{\prime}$ in $S^{\prime}$.

Proof Set $\ell=\operatorname{dim} S$. Then $\operatorname{dim} U=d+\ell+1$ and $\operatorname{dim}\left(S \cap S^{\prime}\right)=\ell-d-1 \geq-1$, so that $\ell \geq d$. We further compute $\operatorname{dim} S^{\prime \prime}=\operatorname{dim} S-\operatorname{dim}\left(S \cap S^{\prime}\right)-1=d$. Let $p \in T^{\prime}$ be arbitrary. Then $\left\langle p, S^{\prime \prime}\right\rangle$ has dimension $d+1$ and hence intersects $S^{\prime}$ in a unique point $p^{\prime}$; the line $\left\langle p, p^{\prime}\right\rangle$ in $\left\langle p, S^{\prime \prime}\right\rangle$ intersects $S^{\prime \prime}$ in a unique point $p^{\prime \prime}$. The map $p \mapsto p^{\prime}$ is a projectivity and hence varying $p$ over $T^{\prime}$, the point $p^{\prime}$ moves across a subspace $S^{\prime \prime \prime}$ of dimension $d$ disjoint from $S \cap S^{\prime}$. The lines $\left\langle p, p^{\prime}\right\rangle$ form a Segre Variety $\mathscr{S}$ of type $(1, d)$. 
Now let $L$ be a line of $U$ intersecting all of $S, S^{\prime}, T^{\prime}$. If $L \cap S \cap S^{\prime} \neq \emptyset$, then $L$ is contained in $C$. Otherwise, let $r, p, p^{\prime}$ be the respective distinct intersections of $L$ with $T^{\prime}, S, S^{\prime}$, and let $M=\left\langle q, q^{\prime}\right\rangle$ (with $q \in S^{\prime \prime}$ and $q^{\prime} \in S^{\prime \prime \prime}$ ) be the unique line in $\mathscr{S}$ through $r$, see the above construction. The lines $M$ and $L$ are contained in a plane and hence the lines $\langle q, p\rangle$ and $\left.q^{\prime} \cdot p^{\prime}\right\rangle$ intersect each other in a point $s \in S \cap S^{\prime}$. So $L \subseteq\langle s, M\rangle \subseteq C$.

The set $C$ of the previous lemma is referred to as a Segre cone (with vertex $S \cap S^{\prime}$ ).

Lemma 5.10 Let $\theta$ be a linear collineation of the projective $\mathbb{K}$-space $\mathbb{P}(V)$ of dimension $n+$ $m$, with $n \geq 0$ and $n+m \geq 3$. Let $S$ be a subspace of $\mathbb{P}(V)$ of dimension $n$ and let $T$ be complementary to $S$ in $\mathbb{P}(V)$, with the property that $T^{\theta} \cap S=\emptyset$. Let $\theta_{T}$ be the map from $T$ to itself given by sending the point $t \in T$ to the unique point of $T \cap\left\langle S, t^{\theta}\right\rangle$. Then $\theta_{T}$ is well-defined and is a linear collineation of $T$. Set $S^{\prime}=S^{\theta^{-1}}, U=\left\langle S, S^{\prime}\right\rangle$ and $T^{\prime}=T \cap U$. Let $C$ be the Segre cone in $U$ with vertex $S \cap S^{\prime}$ containing $S, S^{\prime}$ and $T^{\prime}$. Then the set $P$ of points $p$ of $\mathbb{P}(V)$ with the property that $(\langle S, p\rangle \cap T)^{\theta_{T}}=\left\langle S, p^{\theta}\right\rangle \cap T$ is precisely $\left(C \cup\left\langle S \cap S^{\prime}, T\right\rangle\right) \backslash\left(S \cup S^{\prime}\right)$. In particular, if $S \neq S^{\theta}$, then either $P$ is contained in $C$ (if $U=\mathbb{P}(V)$ ) or in the union of two proper subspaces, namely $P \subseteq\left\langle S \cap S^{\prime}, T\right\rangle \cup U$ (if $W \neq \mathbb{P}(V)$ ).

Proof The map $\theta_{T}$ is well-defined since no point of $T$ is mapped into $S$. Also, as $\theta_{T}$ is the composition of the restriction of $\theta$ to $T$ and a projection, it is a linear collineation of $T$.

Let $p$ be an arbitrary point not contained in $S \cup S^{\prime}$. Since $T \subseteq P$ we may assume $p \notin T$. None of $p$ and $p^{\theta}$ is contained in $S$. Hence $\langle S, p\rangle \cap T$ and $\left\langle S, p^{\theta}\right\rangle \cap T$ are well-defined points, say $p_{T}$ and $p_{T}^{\prime}$. Moreover $p_{T}^{\theta_{T}}=p_{T}^{\prime}$ if and only if $p_{T}^{\theta} \in\left\langle S, p^{\theta}\right\rangle$. This happens if and only if the line $\left\langle p_{T}^{\theta}, p^{\theta}\right\rangle$ (well-defined since we assume $p \neq p_{T}$ ) is contained in $\left\langle S, p^{\theta}\right\rangle$, which is equivalent to $L:=\left\langle p_{T}, p\right\rangle \subseteq\left\langle S^{\prime}, p\right\rangle$. If $p \notin\left\langle S, S^{\prime}\right\rangle$, then this is equivalent with $p \in\left\langle S \cap S^{\prime}, T\right\rangle$. If $p \in\left\langle S, S^{\prime}\right\rangle$, then there are again two possibilities.

- The line $L$ intersects $S \cap S^{\prime}$. In this case $p$ is automatically in $P$. Hence $\left\langle S \cap S^{\prime}, T\right\rangle \backslash S \subseteq P$.

- The line $L$ intersects $S$ and $S^{\prime}$ in distinct points. Since $L$ also intersects $T$, necessarily in a point of $T^{\prime}$, the assertion $p \in P$ is in this case equivalent with $p$ belonging to the Segre cone in $W$ with vertex $S \cap S^{\prime}$ containing $S, S^{\prime}$ and $T^{\prime}$.

The lemma is proved.

Recall that we denote by $G$ the little projective group of $\mathscr{V}_{n}(\mathbb{K}, \mathbb{A})$, and by $G^{\dagger}$ the group of extension of the elements of $G$ to the ambient projective space $\mathbb{P}(V)$ of $\mathscr{V}_{n}(\mathbb{K}, \mathbb{A})$. The next theorem is the main accomplishment of this subsection, and it completes the proof of Main Result 2 for $|\mathbb{K}|>2$. For $n=2$, we also allow $\mathbb{A}$ to be an octonion division algebra over $\mathbb{K}$. For the precise definition of the corresponding standard global Veronesean cap $\mathscr{V} / 2(\mathbb{K}, \mathbb{A})$ we refer to [9].

Theorem 5.11 Suppose we are not in the case $\mathbb{A}=\mathbb{K}=\mathbb{F}_{2}$. Let $\rho$ be the injective projection of $\mathscr{V}_{n}(\mathbb{K}, \mathbb{A}), n \geq 2$, from a subspace $S$ of $\mathbb{P}(V)$ onto a complementary subspace $R$. Denote by $\dagger: G \rightarrow G^{\dagger}$ the natural isomorphism between the little projective group of $\mathscr{V}_{n}(\mathbb{K}, \mathbb{A})$ and the 
group of collineations of $\mathbb{P}(V)$ inducing $G$ on $\mathscr{V}_{n}(\mathbb{K}, \mathbb{A})$ (cf. Proposition 5.1). Let $g \in G$ and denote

$$
g^{\rho}:\left(\mathscr{V}_{n}(\mathbb{K}, \mathbb{A})\right)^{\rho} \rightarrow\left(\mathscr{V}_{n}(\mathbb{K}, \mathbb{A})\right)^{\rho}: p^{\rho} \mapsto p^{g \rho},
$$

where $p \in \mathscr{V}_{n}(\mathbb{K}, \mathbb{A})$. Then $g^{\rho}$ is induced by a collineation of the quotient projective space $\mathbb{P}(V) / S$ if and only if $g^{\dagger}$ stabilises $S$. Hence,

- any G-homogeneous injective image of the standard local Veronesean cap is either the cap itself, or the projection from its non-trivial nucleus space;

- each G-homogeneous local Veronesean cap $\left(X^{\prime}, \Xi^{\prime}\right)$ with $\mathscr{P}\left(X^{\prime}, \Xi^{\prime}\right)=\mathscr{P}^{n}(\mathbb{A})$ is projectively equivalent with $\mathscr{V}_{n}(\mathbb{K}, \mathbb{A})$.

Proof Let $g \in G$. Let $\mathscr{V}_{n}(\mathbb{K}, \mathbb{A})$ have point set $X$. Since $X$ generates $\mathbb{P}(V)$, it contains a basis $B$ of $\mathbb{P}(V)$. Then $B^{\rho}$ contains a basis $B^{\prime \rho}$ of the image projection space, with $B^{\prime} \subseteq B$. We set $T=\left\langle B^{\prime}\right\rangle$. Then $S$ and $T$ are complementary. We can choose $R=T$, i.e., we may assume $b^{\rho}=b$, for all $b \in B^{\prime}$. From now on, we denote by $X^{\prime}$ the image of the projection of $\mathscr{V}_{n}(\mathbb{K}, \mathbb{A})$ from $S$ onto $T$. If $T^{g^{\dagger}} \cap S \neq \emptyset$, then $g^{\rho}$ maps the independent set $B^{\prime}$ of points onto a dependent set of points (since $\operatorname{dim}\left\langle S, T^{g^{\dagger}}\right\rangle<N$ ), and so $g^{\rho}$ has no projective extension in $T$. Note that $S \neq S^{g^{\dagger}}$ in this case.

Hence we may assume that $T^{g^{\dagger}} \cap S=\emptyset$. We now suppose for a contradiction that $S \neq S^{g^{\dagger}}$ and that there is a linear collineation $h$ of $T$ such that its restriction to $X^{\prime}$ yields $g^{\rho}$ (the 'if'-part of the assertion being trivial). We want to apply Lemma 5.10 with $\theta=g^{\dagger}$. Since $\langle S, p\rangle \cap T=p^{\rho}$, for all $p \in T$, we have $\theta_{T}=g^{\dagger} \rho$. We first claim that $g^{\dagger} \rho$ coincides with $h$ over the whole of $T$. In order to prove this, we start by showing that, given any pair of distinct points $p, q \in B^{\prime}$, each point $r \in\langle p, q\rangle$ satisfies $r^{h}=r^{g^{\dagger} \rho}$.

Indeed, for $r \in\{p, q\}$, that is really the definition (since $g$ and $g^{\dagger}$ coincide over $X$ ), hence suppose $r \notin\{p, q\}$. Let $O$ be the $X$-ovoid containing $p, q$.

- If $\langle O\rangle \cap S \neq \emptyset$, then the line $\langle p, q\rangle$ contains at least three images of $X$ under $\rho$, and the result follows from the fact that $g^{\dagger}$ is linear and a linear map on a projective line is determined by three images.

- Suppose now $\langle O\rangle \cap S=\emptyset$. Then, similarly as above, $\left\langle O^{g}\right\rangle \cap S=\langle O\rangle^{g^{\dagger}} \cap S=\emptyset$. Hence, since for each point $x \in O$ we have $x^{\rho g^{\rho}}=x^{g \rho}$, the map $g^{\rho}: O^{\rho} \rightarrow O^{g \rho}$ defines a unique projectivity from $\langle O\rangle^{\rho}$ to $\langle O\rangle^{g^{\dagger} \rho}$ and the image of any point $z^{\rho}$ of $\langle O\rangle^{\rho}$, with $z \in\langle O\rangle$, is given by $z^{g^{\dagger} \rho}$, by uniqueness of $g^{\dagger}$ given $g$. If in particular $z=r \in T$, then $r^{h}=r^{g^{\dagger} \rho}$.

Now this implies that $h$ coincides with $g^{\dagger} \rho$ over a basis and all points of all lines joining any two basis points. This determines $h$ uniquely, and since $g^{\dagger} \rho=\theta_{T}$ is a projectivity (cf. Lemma 5.10), we have that $h$ coincides with $g^{\dagger} \rho$ over the whole of $T$. The claim is proved.

Set $S^{\prime}=S^{\left(g^{\dagger}\right)^{-1}}$. Applying Lemma 5.10 with $\theta=g^{\dagger}$ we claim that $X \subseteq P$, with $P=\{p \in$ $\left.\mathbb{P}(V) \backslash\left(S \cup S^{\prime}\right): p^{\rho g^{\dagger} \rho}=p^{g^{\dagger} \rho}\right\}$ as in Lemma 5.10. Indeed, by definition of $h$, we have for each $x \in X$ that $x^{\rho h}=x^{\rho g^{\rho}}$, and the definition of $g^{\rho}$ then yields $x^{\rho g^{\rho}}=x^{g \rho}$, which equals $x^{g^{\dagger} \rho}$ by the definition of $g^{\dagger}$. By our previous claim, we know $x^{\rho h}=x^{\rho g^{\dagger} \rho}$. The claim is proved. 
Consequently, Lemma 5.10 implies that $X$ is contained in either the union of the two proper subspaces $\left\langle S, S^{\prime}\right\rangle$ and $\left\langle S \cap S^{\prime}, T\right\rangle$, or in a Segre cone $C$ with vertex $S \cap S^{\prime}$. We show that none of this is possible under the given assumptions.

- Suppose $J=\left\langle S, S^{\prime}\right\rangle$ and $K=\left\langle S \cap S^{\prime}, T\right\rangle$ are proper subspaces and $X \subseteq J \cup K$. Since $X$ spans $\mathbb{P}(V)$, there exist a point $x_{1} \in X \cap(J \backslash K)$ and a point $x_{2} \in X \cap(K \backslash J)$. Let $c$ be a conic in $X$ containing $x_{1}$ and $x_{2}$. Then the plane $\pi$ spanned by $c$ intersects $J$ and $K$ in proper subspaces of $\pi$. Hence $c$ is contained in the union of two proper subspaces of $\pi$, which each contain at most 2 points of $c$. Hence $|c| \leq 4$, implying $|\mathbb{K}| \in\{2,3\}$ and $|\mathbb{A}| \in\left\{|\mathbb{K}|,|\mathbb{K}|^{2}\right\}$.

First let $|\mathbb{A}|=|\mathbb{K}|^{2}=9$. Then, in the argument of the previous paragraph, we consider the ovoid $O=X\left(\left[x_{1}, x_{2}\right]\right)$. Then similarly as above, $O$, which contains 10 points, is the union of two proper subspace sections, which each contain at most 4 points (the number of points on a conic), a contradiction.

Set $N=N(n, k)=\operatorname{dim} \mathbb{P}(V)$. Now we show that, if $(n,|\mathbb{A}|) \neq(2,|\mathbb{K}|)$, then $\operatorname{dim} J+$ $\operatorname{dim} K \leq 2 N-4$. Indeed, suppose for a contradiction that $\operatorname{dim} J+\operatorname{dim} K \geq 2 N-3$. Since $\operatorname{dim} J \leq N-1$ and $\operatorname{dim} K \leq N-1$, we have $\operatorname{dim} J, \operatorname{dim} K \in\{N-1, N-2\}$. Set $\operatorname{dim} K=$ $N-a, a \in\{1,2\}$, and $\operatorname{dim} J=N-b, b \in\{1,2\}$, with $a+b \in\{2,3\}$. Then, since $S$ and $T$ are complementary, $\operatorname{dim}\left(S \cap S^{\prime}\right)+a=\operatorname{dim} S$ and so $\operatorname{dim}\left\langle S, S^{\prime}\right\rangle-a=\operatorname{dim} S$, leading to $\operatorname{dim} S=N-a-b$. Hence, in this case, $T$ has dimension $a+b-1$ and so it is either a line or a plane, By the injectivity of $\rho$ that we assume, $|X| \leq|\mathbb{K}|^{2}+|\mathbb{K}|+1$, a contradiction for $(n,|\mathbb{A}|) \neq(2,|\mathbb{K}|)$. Note that, if $a=b=1$, then $|X| \leq|\mathbb{K}|+1$, a contradiction in all cases.

Now let $|\mathbb{A}|=|\mathbb{K}|=3$. By the last line of the foregoing paragraph we may assume that one of $J, K$ is not a hyperplane, say $\operatorname{dim} J \leq N-2$ (the reasoning if $\operatorname{dim} K \leq N-$ 2 is completely the same). By extending $K$ if necessary, we may assume that $K$ is a hyperplane. Then $Q:=K \cap X$ is a quadric in $\mathscr{P}$. Suppose $Q$ contains a conic; then it is easily checked that every point not contained in the vertex of $Q$ (the vertex is the intersection of all maximal subspaces of $Q$ in $\mathscr{P}$ ) is contained in a plane $\pi$ of $\mathscr{P}$ with the property that $\pi \cap Q$ is not the union of two lines in $\pi$. But then the complement of $\pi \cap Q$ in $\pi$ is not contained in any conic of $\pi$ (not even in a degenerate one) and so all points of $\pi$ must belong to $J$. Hence $J$ contains all points of $X$ except for the vertex, a contradiction since this set is not contained in a quadric. We have shown that $Q$ does not contain a conic, hence $Q$ is the union of two hyperplanes $H_{1}$ and $H_{2}$ of $\mathscr{P}$. Then $J$ contains all points off $H_{1} \cup H_{2}$. We claim that $J$ also contains $H_{1} \cap H_{2}$. Indeed, let $L$ be a line of $\mathscr{P}$ intersecting $H_{1} \cap H_{2}$ in a unique point $x$ and not contained in $H_{1} \cup H_{2}$. Then the three points of $L \backslash\left(H_{1} \cap H_{2}\right)$ generate a plane of $\mathbb{P}(V)$ which entirely contains $L$. Hence the claim. Consequently $J$ contains $\left(X \backslash\left(H_{1} \cup H_{2}\right)\right) \cup\left(H_{1} \cap H_{2}\right)$, which is the union of two hyperplanes of $\mathscr{P}$. Hence $J$ is a hyperplane of $\mathbb{P}(V)$ after all, a contradiction.

Finally let $|\mathbb{A}|=|\mathbb{K}|^{2}=4$. Then, completely similar to the previous paragraph, we may assume that $K$ is a hyperplane, $K \cap X$ is the union of three hyperplanes $H_{1}, H_{2}, H_{3}$ of $\mathscr{P}$ with $H_{1} \cap H_{2}=H_{2} \cap H_{3}=H_{3} \cap H_{1}$, and $J$ is generated by the points of the union of the two hyperplanes $H_{4}, H_{5}$ of $\mathscr{P}$ completing $H_{1}, H_{2}, H_{3}$ to a pencil of hyperplanes. Since 
both $H_{4}$ and $H_{5}$ induce local Veronesean caps of index $n-1$, and $\left\langle H_{4}\right\rangle \cap\left\langle H_{5}\right\rangle \cap X$ is local Veronesean cap of index $n-2$, we deduce $\operatorname{dim} J \geq \operatorname{dim}\left\langle H_{4}\right\rangle+\operatorname{dim}\left\langle H_{5}\right\rangle-\operatorname{dim}\left(\left\langle H_{4}\right\rangle \cap\right.$ $\left.\left\langle H_{5}\right\rangle\right)=n^{2}+2 n-2=\operatorname{dim} \mathbb{P}(V)-2$. We also deduce that, if $\operatorname{dim} J=N-2$, then $J \cap X$ is the union of two hyperplanes of $\mathscr{P}$. This immediately implies that one of $J, K$ is a hyperplane of $\mathbb{P}(V)$, and this then contradicts $\operatorname{dim} J+\operatorname{dim} K \leq 2 N-4$.

- Suppose now $X \subseteq C$. Since $X$ admits an injective projection from $S$, it also admits an injective projection $\rho^{\prime \prime}$ from $S \cap S^{\prime}$. The image $X^{\prime \prime}$ of $X$ under $\rho^{\prime \prime}$ is contained in a Segre variety $\mathscr{S}$ of type $(1, d)$, for some positive integer $d$. Since $X$ spans $\mathbb{P}(V)$, we find two points $p, q$ of $X^{\prime \prime}$ in different $d$-spaces of $\mathscr{S}$. These points are not on a common generator of $\mathscr{S}$ as the projection from $S$ is also injective. But then it is easy to see that $\langle p, q\rangle \cap \mathscr{S}=$ $\{p, q\}$. Hence $S \cap S^{\prime}$ is disjoint from $\langle O\rangle$, with $O$ the unique $X$-ovoid through $p$ and $q$. So, considering any plane conic $D$ on $O$ containing $p, q$, we see that $\mathscr{S}$ must contain a conic through $p$ and $q$. However, it is easily calculated that any plane through $p$ and $q$ either intersects $\mathscr{S}$ in a pair of lines, one of which is a generator of $\mathscr{S}$ and hence cannot contain two points of $X$, or has only three points in common with $\mathscr{S}$. Hence $\mathbb{K}=\mathbb{F}_{2}$. Then $\mathscr{S}$ is the union of three $d$-spaces, two of which correspond to $S$ and $S^{\prime}$ and hence which do not contain any point of $X^{\prime \prime}$. Hence $X^{\prime \prime}$ is contained in a $d$-space, contradicting the fact that $X$ spans $\mathbb{P}(V)$ and the projection of $X$ from $S \cap S^{\prime}$ spans a subspace of dimension $2 d+1$.

Now taking into account Propositions 5.4 and 5.5, the theorem is proved.

If $|\mathbb{A}|=|\mathbb{K}|=2$, then the method of proof in Theorem 5.11 fails. However, we believe the result is still true. We can easily show the most important global part of it, which finished the proof of Main Result 2.

Corollary 5.12 Let $G$ be the little projective group of $\mathscr{P}$. Each G-homogeneous local Veronesean cap of index $n, n \geq 2$, is projectively equivalent with $\mathscr{V}_{n}(\mathbb{K}, \mathbb{A})$.

Proof In view of Theorem 5.11 we only need to show this in the case $\mathbb{K}=\mathbb{A}=\mathbb{F}_{2}$.

Let $X$ be the point set of a $G$-homogeneous local Veronesean cap. Suppose first that some hyperplane $Y$ of $\mathscr{P}$ is not full and let $x \in\langle Y\rangle$ be a point of $\mathscr{P}$ off $Y$. Let $g \in G$ be an elation of $\mathscr{P}$ with axis $Y$. Then $g$ fixes $Y$ pointwise and hence the corresponding collineation $g^{\dagger}$ of $\langle X\rangle$ fixes $\langle Y\rangle$ pointwise (as it fixes a generating point set and $\mathbb{K}=\mathbb{F}_{2}$ ), a contradiction, as $g^{\dagger}$ then also fixes $x$, whereas $g$ does not fix $x$. We conclude that every hyperplane is full.

Hence, if $X$ is not projectively equivalent with $\mathscr{V}_{n}(\mathbb{K}, \mathbb{A})$, then by Lemma 4.13 , there is an $X$ conic $C$ not contained in some hyperplane $Y$ of $\mathscr{P}$ such that the nucleus of $X$ is contained in $\langle Y\rangle$. Hence $\langle C\rangle \cap\langle Y\rangle$ is a line $L$. Any elation of $\mathscr{P}$ with axis $Y$ not fixing $C$ extends in $\langle X\rangle$ to a collineation fixing $\langle Y\rangle$ pointwise and mapping $C$ to some $X$-conic $C^{\prime}$ with $L \subseteq\left\langle C^{\prime}\right\rangle$. Hence $\langle C\rangle \cap\left\langle C^{\prime}\right\rangle \supseteq L$, contradicting Axiom (V2).

The corollary is proved.

Remark 5.13 One might wonder what the full automorphism group $\bar{G}$ is of a local standard Veronesean cap. It is beyond the scope of this paper to answer this in detail, but Lemma 4.1 
implies that, for $|\mathbb{K}|>2$, the group $\bar{G}$ is contained in the full collineation group of the associated projective space $\mathscr{P}^{n}(\mathbb{A})$. If $|\mathbb{K}|=2$, then it is not hard to show that, if $|\mathbb{A}|=2$, no seven points of $X$ are contained in a 5-space, except if these seven points form a plane of $\mathscr{P}^{n}(\mathbb{A})$. Hence, if $n \geq 3$, the elliptic spaces are determined by the intersections of appropriate pairs of 5-spaces each spanned by seven points. If $n=2$, then the full symmetric group on seven letters acts on the corresponding global Veronesean cap and is induced by the full collineation group of the ambient projective space $\mathbb{P}(V)$. If $|\mathbb{A}|=4$, then one shows that the only 3 -spaces that contain five points of $X$ are the elliptic spaces. Hence in all cases, except if $|\mathbb{K}|=|\mathbb{A}|=n=2$, the group $\bar{G}$ is contained in the full collineation group of $\mathscr{P}^{n}(\mathbb{A})$ (and we actually conjecture it coincides with it).

If $\mathbb{A}$ is a purely inseparable field extension of $\mathbb{K}$ or $\mathbb{K}=\mathbb{A}$ and char $\mathbb{K}=2$, then for all $n \geq 2$, there is a unique nontrivial subspace stabilised by $G$ and that is the nucleus subspace. We end this paper with the descriptions of the corresponding $G$-homogeneous projection and the geometric structure of the nucleus subspace.

Proposition 5.14 Suppose that $\mathbb{A}$ is a purely inseparable field extension of $\mathbb{K}$ or $\mathbb{K}=\mathbb{A}$ and char $\mathbb{K}=2$. Then the projection of $\mathscr{V}$ from $\mathrm{N}(\mathscr{V})$ is projectively equivalent to the standard embedding of $\mathbb{P}^{n}\left(\mathbb{A}^{2}\right)$ into $\mathbb{P}^{n}(\mathbb{K})$ given by restricting coordinates. (Here, $\mathbb{A}^{2}$ denotes the field of squares of $\mathbb{A}$.)

Proof This follows from the fact that, by the proof of Lemma 5.3, and with the notation of Subsection 2.2, $\mathrm{N}(\mathscr{V})$ consists of the projective points $\left(y_{i}, y_{j, \ell}\right)_{0 \leq i \leq n, 0 \leq j<\ell \leq n}$, with $y_{i}=0$ for all $i \in\{0,1, \ldots, n\}$. Indeed, the projection of an arbitrary point $\mathbb{K}\left(x_{i}^{2} ; x_{j} x_{\ell}\right)_{0 \leq i \leq n, 0 \leq j<\ell \leq n}$ of $\mathscr{V}$ onto the obvious complementary subspace of $\mathrm{N}(\mathscr{V})$ is given by $\left(x_{0}^{2}, x_{1}^{2}, \ldots, x_{n}^{2} ; 0,0, \ldots, 0\right)$.

Proposition 5.15 Let $\mathfrak{P}$ be the set of nuclei spaces of all $X$-ovoids. Then every member of $\mathfrak{P}$ is contained in $\mathrm{N}(\mathscr{V})$. Also, if two members $N_{1}, N_{2} \in \mathfrak{P}$ correspond to intersecting elements $\xi_{1}, \xi_{2}$ of $\Xi$, respectively, then the members of $\mathfrak{P}$ contained in $\left\langle N_{1}, N_{2}\right\rangle$ constitute a partition (spread) $\mathscr{S}$ of $\left\langle N_{1}, N_{2}\right\rangle$. Moreover, a member $N \in \mathfrak{P}$ is contained in $\left\langle N_{1}, N_{2}\right\rangle$ if and only if the corresponding member $\xi$ of $\Xi$ contains $\xi_{1} \cap \xi_{2}$ and is contained in the global Veronesean cap generated by $\xi_{1}, \xi_{2}$.

If we denote by $\mathfrak{L}$ the set of such spreads, then $(\mathfrak{P}, \mathfrak{L})$ with natural incidence defines the pointline space naturally associated with the line-Grassmannian of $\mathscr{P}$.

Proof We use the notation of Subsection 2.2. In order to prove the first assertion, we note that, since $G^{\dagger}$ acts transitively on the family of $X$-ovoids, and since $\mathrm{N}(\mathscr{V})$ is stable under the action of $G^{\dagger}$, it suffices to show that the nucleus space of at least one $X$-ovoid is contained in $\mathrm{N}(\mathscr{V})$. We consider the $X$-ovoid corresponding with the line $(*, *, 0, \ldots, 0)$ of $\mathscr{P}$. Its nucleus subspace is the space defined by $y_{i}=0$, for every $i \in\{0,1, \ldots, n\}$, and $y_{i j}=0$, for every $i$ and $j, i<j, i, j \in\{0,1, \ldots, n\}$, except for $(i, j)=(0,1)$. The first assertion follows. From our argument follows in particularly that the nucleus space of an $X$-ovoid $O$ has dimension $2^{\ell}-1$, and that it is the intersection of $\langle O\rangle$ with $\mathrm{N}(\mathscr{V})$. We now write down what the nucleus subspace of a general $X$-ovoid $O$ looks like. Let the corresponding line of $\mathscr{P}$ be given by the points 
$p=\mathbb{A}\left(x_{0}, x_{1}, \ldots, x_{n}\right)$ and $q=\mathbb{A}\left(y_{0}, y_{1}, \ldots, y_{n}\right)$, and let $p^{\prime}, q^{\prime}$ be the corresponding points of $O$. Then every point of the nucleus of $O$ is the nucleus of a conic $C \subseteq O$, with $\left\{p^{\prime}, q^{\prime}\right\} \subseteq C$, and hence is obtained by intersecting an arbitrary plane through $p^{\prime}$ and $q^{\prime}$ with $\mathrm{N}(\mathscr{V})$. Such an arbitrary plane will contain at least one additional member of $O$, for which we can take the point $r^{\prime}$ on $O$ corresponding to $r=\mathbb{A}\left(k x_{i}+\ell y_{i}\right)_{0 \leq i \leq n}, k, \ell \in \mathbb{A}$. If we just add the coordinates of $p^{\prime}, q^{\prime}$ and $r^{\prime}$ as given in Subsection 2.2, then we obtain

$$
\mathbb{K} k \ell(\underbrace{0,0, \ldots, 0}_{n+1 \text { zeros }}, x_{i} y_{j}+x_{j} y_{i})_{0 \leq i<j \leq n}
$$

Hence, if we reinterpret the subspace of $V$ containing the $\frac{n(n+1)}{2}$ direct factors isomorphic to $\mathbb{A}$ in the definition of $V$ as a projective space over $\mathbb{A}$, then each element of $\mathfrak{P}$ corresponds with a unique point of the line Grassmannian of $\mathscr{P}$ via the Plücker coordinates, in the standard way. Now all assertions follow from the standard properties of Grassmannians.

It now also follows that the action of $G^{\dagger}$ on $\mathrm{N}(\mathscr{V})$ is induced by the natural action of $\mathrm{SL}_{n}(\mathbb{A})$ on the vector space defined by the Plücker coordinates of the vector space over $\mathbb{A}$ underlying $\mathscr{P}$, but considered over $\mathbb{K}$ (also in the natural way). This is completely similar to Proposition 7.2 of [9].

\section{References}

[1] J. André, Über nicht-Desarguessche Ebenen mit transitiver Translationsgruppe, Math. Z. 60 (1954), 156186.

[2] R. Baer, Linear Algebra and Projective Geometry, Academic Press Inc., New York, 1952.

[3] R. H. Bruck \& R. C. Bose, The construction of translation planes from projective spaces, J. Algebra 1 (1964), 85-102.

[4] B. Cooperstein, J. A. Thas \& H. Van Maldeghem, Hermitian Veroneseans over finite fields, Forum Math. 16 (2004), 365-381.

[5] P. Dembowski, Finite Geometries, Ergebnisse der Mathematik und ihrer Grenzgebiete, Band 44. SpringerVerlag, Berlin-New York, 1968.

[6] J. W. P. Hirschfeld \& J. A. Thas, General Galois Geometries, Oxford University Press, 1993.

[7] D. R. Hughes \& F. C. Piper, Projective Planes, Graduate Texts in Mathematics, Vol. 6. Springer-Verlag, New York-Berlin, 1973.

[8] O. Krauss, Geometrische Charakterisierung von Veronesemannigfaltigkeiten. PhD Thesis, Braunschweig, February 2014.

[9] O. Krauss, J. Schillewaert and H. Van Maldeghem, Veronesean representations of Moufang planes, Michigan Math. J. 64 (2015), 819-847.

[10] A. Pasini \& H. Van Maldeghem, Some constructions and embeddings of the Tilde Geometry, Note di Matematica 21 (2002-2003), 1-33.

[11] F. Mazzocca \& N. Melone, Caps and Veronese varieties in projective Galois spaces, Discrete Math. 48 (1984), 243-252.

[12] J. Schillewaert \& H. Van Maldeghem, Hermitian Veronesean caps, in Buildings, Finite Geometries and Groups (ed. N.S. N. Sastry), Springer Proceedings in Mathematics 10 (2012), 175-191.

[13] J. Schillewaert \& H. Van Maldeghem, Quadric Veronesean caps, Bull. Belgian Math. Soc. Simon Stevin 20 (2013), 19-25.

[14] H. Salzmann, D. Betten, T. Grundhöfer, H. Hähl, R. Löwen and M. Stroppel, Compact Projective planes 21, de Gruyter, Berlin, (1995). 
[15] E. E. Shult, Points and Lines, Characterizing the Classical Geometries, Universitext, Springer-Verlag, Berlin Heidelberg, 2011.

[16] J. A. Thas \& H. Van Maldeghem, Classification of finite Veronesean caps, European J. Combin. 25 (2004), $275-285$.

[17] J. Tits, Ovoïdes à translations, Rend. Math. Appl. (5) 21 (1962), 37-59.

[18] J. Tits \& R. M. Weiss, Moufang Polygons, Springer Monographs in Mathematics, Springer-Verlag, Berlin, 2002.

[19] O. Veblen \& J. Young, Projective Geometry, Vol I+II, Blaisdell Publishing Co. Ginn and Co., New YorkToronto-London, 1965. 